\title{
Prevalence of Non-Communicable Diseases and Associated Factors in South Africa: Evidence from National Income Dynamics Survey, 2008-2017.
}

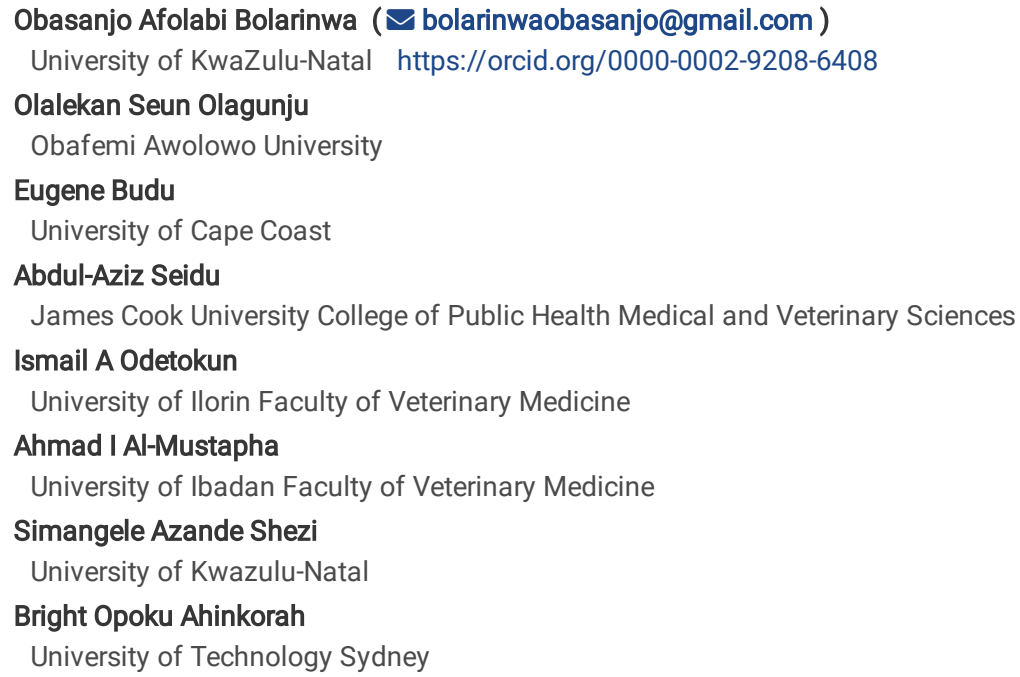




\section{Abstract}

\section{Introduction}

The unprecedented global increase in non-communicable diseases (NCDs) death incidences resulted in a joint initiative by the United Nations (UN) and the World Health Organization (WHO) to reduce NCD-related mortality by $25 \%$ by the year 2025. In this study, we ascertained the prevalence of NCDs in South Africa and its associated factors.

\section{Method}

We used 5-panel waves secondary datasets conducted by national income dynamic survey (NIDS) from 2008 to 2017 among 64,735 South Africans. We calculated the prevalence of all selected NCDs separately and together for each year at the descriptive level. This was followed by the distribution of selected NCDs across the socio-demographic and behavioural characteristics of the respondents. Finally, we used binary logistic regression to assess NCDs' likelihood by the respondents' socio-demographic and behavioural characteristics. The results were presented as crude odds ratios (cOR) and adjusted odds ratios (aOR) with their corresponding $95 \%$ confidence intervals, signifying precision. Statistical significance was set at $p$-value $<0.05$.

\section{Results}

We found that, the prevalence of NCDs in South Africa was highest in the year 2012 (23.8\%) and lowest in the year 2015 (15.2\%). Over the 10-year period, the NCDs with highest and lowest prevalence were high blood pressure and cancer, respectively. Females [cOR $=1.88,95 \% \mathrm{Cl}=1.79-1.96], \mathrm{smokers}$ [cOR=2.01, 95\% $\mathrm{Cl}=1.85-2.19]$ and those very satisfied with life [COR=1.10,95\% $\mathrm{Cl}=1.04-1.17]$ were more likely to have atleast one NCDs. This persisted after adjusting for significant covariates: $[\mathrm{aOR}=1.84,95 \% \mathrm{Cl}=1.74-1.94]$, [aOR=1.49, 95\% $\mathrm{Cl}=1.35-1.65]$ and $[\mathrm{aOR}=1.01,95 \% \mathrm{Cl}=0.95-1.09]$ respectively.

\section{Conclusion}

South Africa's department of health and other health agencies need to strengthen existing policies and develop new interventional frameworks that will deliberately consider various significant factors contributing to the high prevalence of NCDs identified in this study. This will reduce the NCDs prevalence and reduce the morbidity and mortality levels attributable to NCDs in the country.

\section{Introduction}

The increasing incidence of non-communicable diseases (NCDs) such as cardiovascular diseases, cancers, diabetes, and chronic respiratory diseases presents a global health crisis [1]. In 2016, the global NCD-related deaths of 41 million were $14 \%$ higher than the 36 million deaths reported in 2008 [2, 3]. Approximately $81 \%$ of these deaths occurred in low- and middle-income countries (LMICs) [2]. It has been anticipated that NCDs may account for $46 \%$ of subSaharan Africa (SSA) deaths by 2030 [4]. NCD deaths in 23 selected LMICs are estimated to lead to a loss of USD 84 billion in economic production [5, 6]. In 2012, the age-standardized NCD death rate was 539 per 100,000 population globally [3]. The rate was lowest in high-income countries (397 per 100 000) and highest in LMICs $(625$ per 100,000) and SSA (673 per 100,000). Regionally, age-standardized death rates for NCDs ranged from 438 per 100,000 in the WHO Region of the Americas to over 650 per 100,000 in the WHO African, South-East Asia and Eastern Mediterranean Regions [3].

This increasing incidence in NCDs made the United Nations (UN) and the World Health Organization (WHO) to initiate a global call for a $25 \%$ reduction in NCDassociated mortality by 2025 , adopting the slogan " 25 by 25 " [7, 8]. This ' $25 \times 25$ ' strategy focused on six risk factors (tobacco use, excessive alcohol consumption, excessive salt intake, high cholesterol level, hypertension, and obesity) as social determinants of NCDs [9]. By mitigating these risk factors, NCDrelated mortality in South Africa (SA could be reduced by about $20 \%$ [10].

Behavioural risk factors such as tobacco use, unhealthy diet, insufficient physical activity, and excessive alcohol consumption have been associated with increasing NCDs $[11,12]$. Tobacco smoking and exposure to second-hand smoke are responsible for about 6.3 million annual deaths worldwide and $6.3 \%$ of the global burden of disease [13]. In SA, the prevalence of smoking is relatively high. In $2003,35 \%$ of adult men and $10 \%$ of adult women were considered daily or occasional smokers [14]. Smoking is estimated to cause about $71 \%$ of lung cancer, $42 \%$ of chronic respiratory disease and nearly $10 \%$ of cardiovascular diseases [2]

In 2012, an estimated 3.3 million deaths worldwide were attributable to NCDs associated with excessive alcohol consumption, and these accounted for about $3.8 \%$ of all deaths in the world [15]. Cancers, cardiovascular disease and liver cirrhosis were the most frequent alcohol-associated NCDs [16].

The increasing incidence of NCDs has negatively affected SA's sustainable development due to the required health expenditure, disability-adjusted life years (DALYs), and lives lost [5]. SA, with approximately 59 million people, is the most urbanized country in sub-Saharan Africa, with $62 \%$ of its population living in cities [17]. In 2010, NCDs accounted for $39 \%$ of total deaths in SA and 16\% of DALYs [18]. Many of the deaths (36\%) occurred before the age of 60 [19]. Recently, an increase in unhealthy dietary patterns and a decrease in physical activities have resulted in a rising NCD burden in SA [19]. For instance, in SSA, SA has the highest burden of cardiovascular diseases and a very high burden of type 2 diabetes mellitus [20]. The primary health care system in SA is inadequately prepared to meet the increasing health demands posed by NCDs, especially with the recent efforts aimed at up-scaling the antiretroviral therapy for HIV and AIDS patients [20]. The NCD epidemic in SA is further complicated by an aging HIV-positive population [10].

Estimates by Dalal et al. [21] and Solomons et al. [22] showed that NCDs would be responsible for $46 \%$ of deaths in SSA, with higher age-standardized death rates in four of these countries (DRC, Ethiopia, Nigeria, and South Africa) compared to those in high-income countries. NCDs, which are attributable to the above risk factors, hold serious consequences for economic, social, and developmental growth; thus, there is a need to investigate the underlining associated 
risk leading to a high prevalence of NCDs [22]. Hence, this study aimed to determine the prevalence and examine the risk factors associated with NCDs' occurrence among the South African adult population from 2008 to 2017.

\section{Data And Methods}

\section{Description of the study area}

The study was conducted in SA, located in the Southernmost part of Africa with a population of 53.5 million people and constitutes about $8.3 \%$ of Africa's population size [23]. In addition to having one of the highest prevalence of HIV/AIDS globally, SA continues to face high NCDs rates, with $41 \%$ of all deaths being caused by NCDs [24].

\section{The rationale for the study}

Based on previous studies, among the male population, $43 \%$ had raised blood pressure, $46 \%$ were physically inactive [25], while $31 \%$ had raised blood cholesterol [26]. On the other hand, among the female population, $41 \%$ had raised blood level, $56 \%$ were physically inactive, and $37 \%$ had raised blood cholesterol.

In light of this, the government of SA initiated the strategic plan for the prevention of NCDs in 2013 by putting in place a regulatory framework around salt consumption in addition to the intervention to limit access to tobacco with the view to achieving a significant reduction in the prevalence of NCDs and associated mortality [27].

\section{Target population}

The population considered in this study were adults (males and females) between the ages of 18 and above in SA. Respondents who were less than 18 years were excluded from the study.

\section{Data source}

This study extracted the data of both male and female older adults (i.e., at least 18 years and above) in South Africa from the overall data collected from a sample of adults in the National Income Dynamics Study (NIDS). The NIDS is the first nationally representative household-based longitudinal (panel) survey in SA - implemented by the Southern Africa Labour and Development Research Unit (SALDRU) at the University of Cape Town every two years - based on which accurate and reliable estimates of socio-economic (poverty, wellbeing, inequality, education, human capital formation, labour market participation and economic activity, vulnerability and social capital) demographic (fertility, mortality and household composition and migration) and health parameters were derived and routinely monitored [28].

In the first year of the surveys in 2008, a nationally representative sample of 28,000 individuals in 7,300 households was interviewed and followed up to the year 2017. Also, any new members of the sampled household were interviewed but not followed-up. Thus, a total of five surveys have been conducted so far. Being a feature of a panel study, there were attrition cases among the Whites, Indian/Asians, and high-income respondents. So, to reduce the effect of sampling bias and coverage error age in the fifth wave (2017) as a result of attrition, an extra (top-up) sample of 2,775 individuals was added. Hence, all the previously released weights (design, calibrated and panel waves) in waves one to four were recalculated while the current weight in wave (2017) considered the attrition rate and top-up sample inclusion. After considering the study population, the total sample size for this study was 64,735 with each year sample size as follows 2008 was 11,051, 2011 was 13,793, 2012 was 13,753, 2015 was 12,427, and 2017 was 13,712. More information about the study can be obtained from the NIDS website: http://www.nids.uct.ac.za/.

The 2008-2017 datasets were formally requested and obtained from the Department of Planning, Monitoring and Evaluation (DPME) and SALDRU. The datasets have been blinded and therefore pose no threat to any interviewed respondent, households, community or nation.

\section{Variables selection}

\section{Outcome variables}

The study's main outcome variables were high blood pressure, diabetes, asthma, heart problem, cancer, and at least one NCD. Blood pressure, diabetes, asthma, heart problem, and cancer were derived from questions that asked respondents whether they had been diagnosed with any of these diseases. For each of them, the responses were 'yes' or 'no'. Those who answered 'yes' to any of the questions were considered having blood pressure, diabetes, asthma, heart problems, or cancer. Out of these five NCDs, a composite variable 'at least one NCD' was generated and represented respondents who had been diagnosed with either blood pressure, diabetes, asthma, heart problem or cancer.

\section{Explanatory variables}

Ten explanatory variables were considered in this study. The variables were age (18-24, 25-34, 35-44, 45-54, 55-64 and 65+), gender (male and female), employment status (unemployed and employed), marital status (never married, married, living with partner, widow/widower and divorced/separated) and population group (African, colored, Asian/Indian and White). Other explanatory variables were religion (no religion, Christianity, Islam, Traditional religion and others) and education (no formal education, primary, secondary and tertiary). Level of satisfaction with life (dissatisfied, satisfied and very satisfied), alcohol consumption (never, rarely, once a week and every day) and frequency of exercise (never, rarely and once a week) were also considered as explanatory 
variables. These variables were not selected a priori but based on their availability in the datasets and previous studies [29, 30], finding significant factors associated with non-communicable diseases.

\section{Statistical analysis}

Data used in this study were processed and analysed using STATA version 16.0. Both descriptive and inferential statistics were employed. The analysis began using descriptive statistics of frequency and percentages to present the respondents' demographic characteristics for the five survey waves (2008, 2011, 2012, 2015 and 2017). This was followed by the use of bar charts and line graphs to show a graphical representation of the proportions of respondents diagnosed with blood pressure, diabetes, asthma, heart problem, cancer and at least one of these diseases, respectively. Next, the five datasets were appended, and Pearson's chi-square test of independence was used to examine the association between the explanatory variables and the outcome variables. Finally, both bivariate and multivariable binary logistic regression was used to assess the risk factors for blood pressure, diabetes, asthma, heart problem, cancer and at least one of these diseases. Results were presented as crude odds ratios (cORs) and adjusted odds ratios (aORs), with their corresponding $95 \%$ confidence intervals (Cls) signifying their level of precision. Statistical significance was declared at $p<0.05$. Sample weight was applied to cater to under and oversampling across the outcome and explanatory variables $[26,31,32]$.

\section{Results}

\section{Demographic characteristics of the respondents}

Over the 10-year period, majority of the respondents were aged $25-34$ (25.3\% in $2008,25.2 \%$ in $2011,26.5 \%$ in $2012,27.0 \%$ in 2015 and $29.1 \%$ in 2017 , females $(56.4 \%$ in $2008,54.1 \%$ in $2011,54.4 \%$ in $2012,53.3 \%$ in 2015 and $53.4 \%$ in 2017 ), unemployed (68.9\% in $2008,68.9 \%$ in $2011,65.7 \%$ in $2012,62.9 \%$ in 2015 and $61.3 \%$ in 2017) and never married (45.5\% in 2008, 51.5\% in 2011, 52.0\% in 2012, 67.4\% in 2015 and $68.3 \%$ in 2017 ). The results further showed that over the 10-year period, most of the respondents were Africans (78.3\% in $2008,78.8 \%$ in $2011,78.7 \%$ in $2012,79.1 \%$ in 2015 and $81.8 \%$ in 2017 ), Christians ( $82.2 \%$ in $2008,76.1 \%$ in $2011,78.5 \%$ in $2012,82.6 \%$ in 2015 and $79.3 \%$ in 2017 ), had tertiary education $(70.1 \%$ in $2008,74.3 \%$ in $2011,75.6 \%$ in $2012,82.3 \%$ in 2015 and $82.0 \%$ in 2017). Between 2008 and 2017 , most of the respondents were satisfied with life $(48.6 \%$ in $2008,49.6 \%$ in $2011,53.0 \%$ in $2012,57.5 \%$ in 2015 and $56.6 \%$ in 2017), non-smokers (94\% in 2008, 96.6\% in 2011, 96.0\% in 2012, $93.1 \%$ in 2015 and $94.8 \%$ in 2017 ) and never exercised (64.0\% in 2008 , $70.6 \%$ in $2011,69.4 \%$ in $2012,64.6 \%$ in 2015 and $64.8 \%$ in 2017) (Table 1).

Table 1: Socio-demographic and behavioural characteristics of the respondents 


\begin{tabular}{|c|c|c|c|c|c|}
\hline Variables & 2008 & 2011 & 2012 & 2015 & 2017 \\
\hline $\mathrm{N}=64,735$ & $\%$ & $\%$ & $\%$ & $\%$ & $\%$ \\
\hline \multicolumn{6}{|l|}{ Age group } \\
\hline $18-24$ & 22.1 & 21.5 & 20.2 & 19.9 & 18.2 \\
\hline $25-34$ & 25.3 & 25.2 & 26.5 & 27.0 & 29.1 \\
\hline $35-44$ & 19.7 & 20.0 & 20.0 & 19.6 & 20.5 \\
\hline $45-54$ & 14.7 & 14.7 & 14.3 & 14.8 & 14.2 \\
\hline $55-64$ & 10.1 & 10.5 & 10.6 & 10.2 & 9.7 \\
\hline $65+$ & 8.1 & 8.3 & 8.4 & 8.6 & 8.3 \\
\hline Mean age: & 39 years & 39 years & 39 years & 39 years & 39 years \\
\hline \multicolumn{6}{|l|}{ Gender } \\
\hline Male & 43.6 & 45.9 & 45.6 & 46.7 & 46.6 \\
\hline Female & 56.4 & 54.1 & 54.4 & 53.3 & 53.4 \\
\hline \multicolumn{6}{|l|}{ Employment status } \\
\hline Unemployed & 68.9 & 68.9 & 65.7 & 62.9 & 61.3 \\
\hline Employed & 31.1 & 31.1 & 34.3 & 37.1 & 38.7 \\
\hline \multicolumn{6}{|l|}{ Marital status } \\
\hline Never married & 45.5 & 51.5 & 52.0 & 67.4 & 68.3 \\
\hline Married & 33.7 & 31.4 & 29.9 & 27.6 & 25.8 \\
\hline living with partner & 9.9 & 7.8 & 7.6 & 0.2 & 0.5 \\
\hline Widow/widower & 7.4 & 6.5 & 7.2 & 1.1 & 1.1 \\
\hline Divorced/separated & 3.5 & 2.7 & 3.2 & 3.8 & 4.2 \\
\hline \multicolumn{6}{|l|}{ Population group } \\
\hline African & 78.3 & 78.8 & 78.7 & 79.1 & 81.8 \\
\hline Colored & 8.2 & 8.5 & 9.2 & 9.0 & 8.8 \\
\hline Asian/Indian & 2.4 & 2.7 & 2.6 & 2.7 & 2.1 \\
\hline White & 11.1 & 10.2 & 9.8 & 9.5 & 7.6 \\
\hline \multicolumn{6}{|l|}{ Religion } \\
\hline No religion & 10.6 & 13.9 & 11.2 & 7.6 & 11.6 \\
\hline Christianity & 82.2 & 76.1 & 78.5 & 82.6 & 79.3 \\
\hline Islam & 1.1 & 1.0 & 0.9 & 1.3 & 1.1 \\
\hline Traditional religion & 4.6 & 7.4 & 6.8 & 6.7 & 6.6 \\
\hline Others & 1.5 & 1.6 & 2.6 & 1.8 & 1.5 \\
\hline \multicolumn{6}{|l|}{ Education } \\
\hline No formal education & 10.1 & 8.3 & 7.7 & 2.4 & 5.2 \\
\hline Primary & 2.0 & 1.6 & 1.2 & 1.1 & 0.9 \\
\hline Secondary & 17.8 & 15.8 & 15.5 & 14.1 & 11.9 \\
\hline Tertiary & 70.1 & 74.3 & 75.6 & 82.3 & 82.0 \\
\hline \multicolumn{6}{|c|}{ Level of satisfaction with life } \\
\hline Dissatisfied & 30.0 & 33.7 & 29.6 & 21.1 & 21.9 \\
\hline Satisfied & 48.6 & 49.6 & 53.0 & 57.5 & 56.6 \\
\hline Very satisfied & 21.4 & 16.7 & 17.4 & 21.4 & 21.5 \\
\hline \multicolumn{6}{|l|}{ Smoking } \\
\hline No & 94.0 & 96.6 & 96.0 & 93.1 & 94.8 \\
\hline
\end{tabular}




\begin{tabular}{|llllll|} 
Yes & 6.0 & 3.4 & 4.0 & 6.9 & 5.2 \\
\hline How often do you exercise & & & & & \\
\hline Never & 64.0 & 70.6 & 69.4 & 64.6 & 64.8 \\
\hline Rarely & 7.5 & 8.3 & 8.5 & 8.9 & 8.4 \\
\hline Once a week & 28.5 & 21.1 & 22.1 & 26.5 & 26.8 \\
\hline
\end{tabular}

Trends of non-communicable diseases from 2008-2017

Over the 10 years, the highest prevalence of high blood pressure (17.3\%), diabetes (5\%), asthma (4.1\%), heart problem (2.7\%) was recorded in 2012 . However, the highest prevalence of cancer was recorded in 2015 (1.3\%) (Figure 1). In terms of at least one non-communicable disease, the prevalence reduced from $23.0 \%$ to $17.4 \%$ between 2008 to 2011 , increased to $23.8 \%$ in 2012 , reduced further from $23.8 \%$ to $15.2 \%$ in 2015 and increased to $18.9 \%$ in 2017 (Figure 2 ).

Figure 1: Trends of non-communicable diseases from 2008-2017

Figure 2: Trends of at least one non-communicable disease from 2008-2017

\section{Distribution of non-communicable diseases across the socio-demographic and behavioural variables}

The results indicated that most of the study participants who had at least one NCD were aged 65+ (54.0\%), males (13.1\%), whites (36.4\%), widowed (49.3\%), not working (20.0\%), belonged to other religion (25.3\%), had primary education (40.0\%), ever smoked (31.4\%), never exercised (20.8\%) and were very satisfied with life (20.4\%). Details of the distribution of the socio-demographic characteristics across each of the NCDs are shown in Table 2 . The chi-square test results indicated statistically significant associations between the socio-demographic characteristics and the occurrence of blood pressure, diabetes, and at least one NCD. Conversely, exercise and satisfaction level of life showed no statistical significance with asthma. Similarly, exercise had no statistically significant association with heart problems.

Table 2: Distribution of non-communicable diseases across the socio-demographic and behavioural variables 


\begin{tabular}{|c|c|c|c|c|c|c|}
\hline \multicolumn{7}{|l|}{$\mathrm{N}=64,735$} \\
\hline Age & $\%$ & $\%$ & $\%$ & $\%$ & $\%$ & $\%$ \\
\hline $18-24$ & 1.4 & 2.0 & 0.6 & 0.3 & 0.3 & 4.2 \\
\hline $25-34$ & 4.3 & 1.9 & 0.7 & 0.6 & 0.3 & 7.3 \\
\hline $35-44$ & 11.7 & 2.8 & 1.4 & 2.6 & 0.6 & 16.3 \\
\hline $45-54$ & 26.3 & 4.6 & 3.4 & 7.1 & 1.1 & 32.9 \\
\hline $55-64$ & 40.2 & 5.0 & 5.5 & 13.1 & 1.3 & 47.7 \\
\hline $65+$ & 46.0 & 4.9 & 7.1 & 13.5 & 1.8 & 54.0 \\
\hline p-value & $7.5(<0.001)$ & $292.9(<0.001)$ & $6.8(<0.001)$ & $14.1(<0.001)$ & $216.8(<0.001)$ & $6.5(<0.001)$ \\
\hline \multicolumn{7}{|l|}{ Gender } \\
\hline Male & 9.0 & 2.2 & 1.3 & 3.2 & 0.6 & 13.1 \\
\hline Female & 17.3 & 3.4 & 2.6 & 4.3 & 0.8 & 22.1 \\
\hline p-value & $800.8(<0.001)$ & $54.0(<0.001)$ & $104.9(<0.001)$ & $50.7(<0.001)$ & $7.3(<0.001)$ & $755.1(<0.001)$ \\
\hline \multicolumn{7}{|l|}{ Population group } \\
\hline African & 13.1 & 2.7 & 1.8 & 3.5 & 0.5 & 17.2 \\
\hline Coloured & 22.8 & 4.1 & 3.4 & 6.4 & 0.8 & 28.4 \\
\hline Asian/Indian & 19.2 & 6.8 & 5.2 & 10.7 & 0.8 & 27.8 \\
\hline White & 24.1 & 7.1 & 6.1 & 7.4 & 4.5 & 36.4 \\
\hline p-value & $606.8(<0.001)$ & $153.4(<0.001)$ & $280.2(<0.001)$ & $295.2(<0.001)$ & $510.9(<0.001)$ & $949.4(<0.001)$ \\
\hline \multicolumn{7}{|l|}{ Marital status } \\
\hline Married & 25.3 & 3.6 & 3.5 & 7.7 & 1.2 & 31.8 \\
\hline Living with partner & 14.1 & 3.3 & 2.0 & 2.4 & 0.7 & 19.0 \\
\hline Widowed & 42.3 & 4.8 & 7.0 & 12.6 & 1.0 & 49.3 \\
\hline Divorced/separated & 26.4 & 4.5 & 3.5 & 7.6 & 1.7 & 34.2 \\
\hline Never married & 6.8 & 2.4 & 1.1 & 1.5 & 0.4 & 10.2 \\
\hline p-value & $49.7(<0.001)$ & $105.2(<0.001)$ & $817.1(<0.001)$ & $8.7(<0.001)$ & $122.1(<0.001)$ & $20.1(<0.001)$ \\
\hline \multicolumn{7}{|l|}{ Employment status } \\
\hline Not working & 15.4 & 3.1 & 2.4 & 4.3 & 0.7 & 20.0 \\
\hline Working & 11.9 & 2.6 & 1.4 & 3.0 & 0.6 & 16.3 \\
\hline p-value & $128.4(<0.001)$ & $8.1(<0.001)$ & $68.1(<0.001)$ & $62.9(<0.001)$ & $1.9(0.17)$ & $111.2(<0.001)$ \\
\hline \multicolumn{7}{|l|}{ Religion } \\
\hline No religion & 9.5 & 2.6 & 1.5 & 3.0 & 0.5 & 13.5 \\
\hline Christian & 15.2 & 3.1 & 2.2 & 4.0 & 0.7 & 19.8 \\
\hline Muslim & 12.6 & 3.7 & 4.2 & 8.6 & 1.3 & 21.3 \\
\hline Traditional & 12.9 & 2.3 & 1.3 & 3.5 & 0.3 & 16.5 \\
\hline Others & 17.9 & 5.0 & 5.0 & 6.7 & 0.9 & 25.3 \\
\hline p-value & $155.0(<0.001)$ & $14.6(<0.001)$ & $65.1(<0.001)$ & $50.9(<0.001)$ & $16.8(<0.001)$ & $175.4(<0.001)$ \\
\hline \multicolumn{7}{|l|}{ Education } \\
\hline No schooling & 32.6 & 4.1 & 4.0 & 8.8 & 0.6 & 39.0 \\
\hline Primary & 34.0 & 6.3 & 6.1 & 8.8 & 1.0 & 40.0 \\
\hline Secondary & 25.9 & 4.0 & 4.0 & 7.1 & 0.8 & 31.7 \\
\hline Tertiary & 9.2 & 2.5 & 1.4 & 2.5 & 0.7 & 13.2 \\
\hline p-value & $14.1(<0.001)$ & $109.8(<0.001)$ & $468.2(<0.001)$ & $958.9(<0.001)$ & $2.0(0.583)$ & $13.9(<0.001)$ \\
\hline
\end{tabular}




\begin{tabular}{|c|c|c|c|c|c|c|}
\hline \multicolumn{7}{|l|}{ Ever smoked } \\
\hline No & 14.1 & 2.9 & 2.0 & 3.9 & 0.6 & 18.5 \\
\hline Yes & 22.9 & 5.3 & 5.2 & 6.2 & 1.9 & 31.4 \\
\hline p-value & $106.5(<0.001)$ & $41.0(<0.001)$ & $128.0(<0.001)$ & $37.9(<0.001)$ & $57.0(<0.001)$ & $279.1(<0.001)$ \\
\hline \multicolumn{7}{|l|}{ Exercise } \\
\hline Never & 16.2 & 3.1 & 2.2 & 4.3 & 0.6 & 20.8 \\
\hline Rarely & 11.4 & 2.7 & 1.9 & 3.5 & 0.8 & 16.2 \\
\hline Once a week & 9.4 & 2.7 & 2.0 & 2.8 & 0.9 & 13.9 \\
\hline p-value & $437.8(<0.001)$ & $4.2(0.12)$ & $5.0(0.08)$ & $61.6(<0.001)$ & $15.1(<0.001)$ & $345.9(<0.001)$ \\
\hline \multicolumn{7}{|c|}{ Satisfaction level of life } \\
\hline Dissatisfied & 14.4 & 3.0 & 2.3 & 4.0 & 0.7 & 18.9 \\
\hline Satisfied & 14.3 & 2.9 & 2.0 & 3.8 & 0.6 & 18.6 \\
\hline Very satisfied & 15.3 & 3.2 & 2.3 & 4.3 & 1.0 & 20.4 \\
\hline p-value & $7.0\left(0.03^{*}\right)$ & $1.3(0.51)$ & $7.0\left(0.03^{*}\right)$ & $7.4(<0.001)$ & $22.1(<0.001)$ & $19.5(<0.001)$ \\
\hline
\end{tabular}

* $p<0.05$

Table 3 showed that study participants that were aged $65+$ had the highest likelihood of being diagnosed with high blood pressure [cOR=61.04, $95 \% \mathrm{Cl}=53.29$ 69.93], heart problem [COR $=13.05,95 \% \mathrm{Cl}=10.50-16.21$ ], diabetes [UOR=50.93, 95\% $\mathrm{Cl}=38.67-67.10]$, cancer [cOR $=5.51,95 \% \mathrm{Cl}=4.00-7.59]$ and at least one $\mathrm{NCD}[\mathrm{COR}=26.76,95 \% \mathrm{Cl}=24.47-29.27]$, compared to those aged 18-24. However, the highest odds of being diagnosed of Asthma was found among respondents aged 55-64 [COR $=2.65,95 \% \mathrm{Cl}=2.22-3.16]$. Females were more likely to have blood pressure $[\mathrm{cOR}=2.10,95 \% \mathrm{Cl}=2.00-2.22], \mathrm{Asthma}[\mathrm{cOR}=1.55$, $95 \% \mathrm{Cl}=1.38-1.74]$, heart problem [COR $=1.95,95 \% \mathrm{Cl}=1.71-2.22$ ], diabetes [COR $=1.38,95 \% \mathrm{Cl}=1.26-1.50]$, cancer [cOR $=1.33,95 \% \mathrm{Cl}=1.08-1.63$ ] and at least one NCD [COR $=1.88,95 \% \mathrm{Cl}=1.79-1.96$ ] compared to males. There were higher odds of having blood pressure [cOR $=2.11,95 \% \mathrm{Cl}=1.91-2.32]$, Asthma [cOR $=2.76,95 \% \mathrm{Cl}=2.26-3.37$ ], heart problem [COR $=3.53,95 \% \mathrm{Cl}=2.95-4.24]$, cancer [COR =8.90, 95\% $\mathrm{Cl}=7.09-11.18$ ] and at least one $\mathrm{NCD}$ [cOR $=2.76,95 \% \mathrm{Cl}=2.53-$ 3.01] among white South Africans, compared to African South Africans. However, the odds of having diabetes was high among Asian/Indian South Africans [COR=3.35, 95\% Cl=2.66-4.22]. Respondents who were widowed, were more likely to have high blood pressure [cOR =2.16, 95\% $\mathrm{Cl}=2.01-2.32$ ], Asthma [cOR $=1.35,95 \% \mathrm{Cl}=1.14-1.61]$, heart problem [COR $=2.11,95 \% \mathrm{Cl}=1.82-2.45]$, diabetes [COR $=1.73,95 \% \mathrm{Cl}=1.55-1.93$ ] and at least one $\mathrm{NCD}[\mathrm{cOR}=2.09,95 \% \mathrm{Cl}=1.95-$ 2.24], compared to those who were married.

Over the ten year period, respondents who belonged to other religion, were more likely to have high blood pressure [cOR $=2.06,95 \% \mathrm{Cl}=1.67-2.56]$, Asthma [cOR $=1.97,95 \% \mathrm{Cl}=1.26-3.08]$, heart problem [COR $=3.48,95 \% \mathrm{Cl}=2.32-5.21]$ and at least one $\mathrm{NCD}$ [COR $=2.14,95 \% \mathrm{Cl}=1.78-2.59]$, compared to those who had no religion. However, the odds of having diabetes was high among Muslims compared to those with no religion [cOR $=3.09,95 \% \mathrm{Cl}=2.03-4.71]$. There were higher odds of have high blood pressure [cOR $=1.80,95 \% \mathrm{Cl}=1.64-1.98]$, Asthma [COR =2.06, 95\% Cl=1.73-2.45], heart problem [cOR =2.69, 95\% $\mathrm{Cl}=2.25-3.21$ ], diabetes [COR $=1.65,95 \% \mathrm{Cl} 1.40-1.94]$, cancer [UOR=2.94, 95\% Cl=2.19-3.95] and at least one NCD [COR $=2.01,95 \% \mathrm{Cl}=1.85-2.19]$ among respondents who smoked, compared to non-smokers. Respondents who are very satisfied with life were more likely to have high blood pressure [cOR $=1.07,95 \% \mathrm{Cl}=1.01-1.14]$, cancer [COR $=1.54,95 \% \mathrm{Cl}=1.20-1.98$ ] and at least one NCD [COR $=1.10,95 \% \mathrm{Cl}=1.04-1.17]$ compared to those who were dissatisfied with life. Lower odds of high blood pressure [COR $=0.21,95 \% \mathrm{Cl}=0.20-0.22]$, Asthma [COR $=0.61,95 \% \mathrm{Cl}=0.52-0.70]$, heart problem [cOR $=0.35,95 \% \mathrm{Cl}=0.30-0.40]$, diabetes and at least one NCD [COR $=0.24,95 \% \mathrm{Cl}=0.23-0.25]$ were found among respondents with tertiary education, compared to those with no formal education. Respondents who exercised once a week were less likely to have high blood pressure [COR $=0.53,95 \% \mathrm{Cl}=0.50-0.57$ ], cancer [cOR $=0.65,95 \% \mathrm{Cl}=0.58-0.73$ ] and at least one $\mathrm{NCD}[\mathrm{COR}=0.62,95 \% \mathrm{Cl}=0.58-0.65]$, compared to those who never exercised. Conversely, the likelihood of having diabetes increased among respondents who exercised once a week [COR $=1.50,95 \% \mathrm{Cl}=1.22-1.85]$, compared to those who never exercised. In terms of survey years, high prevalence of blood pressure [cOR $=1.11,95 \% \mathrm{Cl}=1.04-1.18]$ and diabetes [ $\mathrm{COR}=1.21,95 \% \mathrm{Cl}=1.08-1.35]$ were recorded in 2012, compared to 2008.

Table 3: Bivariate logistic regression of the association between socio-demographic, behavioural characteristics and non-communicable diseases 


\begin{tabular}{|c|c|c|c|c|c|c|}
\hline Variables & Blood pressure & Asthma & Heart problem & Diabetes & Cancer & At least one NCD \\
\hline \multicolumn{7}{|l|}{$\mathrm{N}=64,735$} \\
\hline & cOR $(95 \% \mathrm{Cl})$ & cOR $(95 \% \mathrm{Cl})$ & cOR $(95 \% \mathrm{Cl})$ & cOR $(95 \% \mathrm{Cl})$ & cOR $(95 \% \mathrm{Cl})$ & cOR $(95 \% \mathrm{Cl})$ \\
\hline \multicolumn{7}{|l|}{ Age } \\
\hline $18-24$ & 1 & 1 & 1 & 1 & 1 & 1 \\
\hline $25-34$ & $3.25^{\star \star \star}(2.80-3.76)$ & $0.97(0.82-1.16)$ & $1.24(0.95-1.61)$ & $1.94^{\star \star \star}(1.39-2.70)$ & $1.03(0.72-1.49)$ & $1.78^{\star \star \star}(1.62-2.00)$ \\
\hline $35-44$ & $9.50^{\star \star \star}(8.28-10.92)$ & $\begin{array}{l}1.43^{\star \star \star}(1.20- \\
1.71)\end{array}$ & $2.48^{\star \star \star}(1.93-3.18)$ & $8.78^{\star \star \star}(6.57-11.74)$ & $\begin{array}{l}1.79^{\star \star \star}(1.26- \\
2.55)\end{array}$ & $4.43^{\star \star \star}(4.06-4.85)$ \\
\hline $45-54$ & $\begin{array}{l}25.53^{\star \star \star}(22.32- \\
29.21)\end{array}$ & $\begin{array}{l}2.42^{\star \star \star}(2.05- \\
2.85)\end{array}$ & $5.99^{\star \star \star}(4.78-7.50)$ & $\begin{array}{l}24.79^{\star \star \star}(18.78- \\
32.73)\end{array}$ & $\begin{array}{l}3.42^{\star \star \star}(2.47- \\
4.73)\end{array}$ & $\begin{array}{l}11.16^{\star \star *}(10.24- \\
12.16)\end{array}$ \\
\hline $55-64$ & $\begin{array}{l}47.89^{\star * \star}(41.82- \\
54.84)\end{array}$ & $\begin{array}{l}2.65^{\star \star \star}(2.22- \\
3.16)\end{array}$ & $\begin{array}{l}10.00^{\star * \star}(8.02- \\
12.48)\end{array}$ & $\begin{array}{l}49.26^{\star \star \star}(37.41- \\
64.85)\end{array}$ & $\begin{array}{l}3.85^{\star \star \star}(2.74- \\
5.40)\end{array}$ & $\begin{array}{l}20.73^{\star \star \star}(18.97- \\
22.64)\end{array}$ \\
\hline $65+$ & $\begin{array}{l}61.04^{\star \star *}(53.29- \\
69.93)\end{array}$ & $\begin{array}{l}2.60^{\star \star \star}(2.18- \\
3.11)\end{array}$ & $\begin{array}{l}13.05^{* \star \star}(10.50- \\
16.21)\end{array}$ & $\begin{array}{l}50.93^{\star \star \star}(38.67- \\
67.10)\end{array}$ & $\begin{array}{l}5.51^{* \star \star}(4.00- \\
7.59)\end{array}$ & $\begin{array}{l}26.76^{\star \star \star}(24.47- \\
29.27)\end{array}$ \\
\hline \multicolumn{7}{|l|}{ Gender } \\
\hline Male & 1 & 1 & 1 & 1 & 1 & 1 \\
\hline Female & $2.10^{\star \star \star}(2.00-2.22)$ & $\begin{array}{l}1.55^{\star \star \star} \\
1.74)\end{array}$ & $1.95^{\star \star \star}(1.71-2.22)$ & $1.38^{\star \star \star}(1.26-1.50)$ & $\begin{array}{l}1.33^{\star \star \star}(1.08- \\
1.63)\end{array}$ & $1.88^{\star \star \star}(1.79-1.96)$ \\
\hline \multicolumn{7}{|l|}{ Population group } \\
\hline African & 1 & 1 & 1 & 1 & 1 & 1 \\
\hline Coloured & $1.96^{\star \star \star}(1.83-2.09)$ & $\begin{array}{l}1.55^{\star \star \star}(1.33- \\
1.81)\end{array}$ & $1.89^{\star \star \star}(1.62-2.21)$ & $1.90^{\star \star \star}(1.70-2.13)$ & $1.45^{*}(1.06-1.98)$ & $1.91^{\star \star \star}(1.80-2.02)$ \\
\hline Asian/Indian & $1.58^{\star \star \star}(1.32-1.89)$ & $\begin{array}{l}2.64^{\star * \star}(1.89- \\
3.69)\end{array}$ & $3.01^{\star \star \star}(2.18-4.14)$ & $3.35^{\star \star \star}(2.66-4.22)$ & $1.45(0.64-3.26)$ & $1.84^{\star \star \star}(1.57-2.15)$ \\
\hline White & $2.11^{\star \star \star}(1.91-2.32)$ & $\begin{array}{l}2.76^{\star \star \star}(2.26- \\
3.37)\end{array}$ & $3.53^{\star \star \star}(2.95-4.24)$ & $2.23^{\star \star \star}(1.90-2.63)$ & $\begin{array}{l}8.90^{\star \star \star}(7.09- \\
11.18)\end{array}$ & $2.76^{\star \star \star}(2.53-3.01)$ \\
\hline \multicolumn{7}{|l|}{ Marital status } \\
\hline Married & 1 & 1 & 1 & 1 & 1 & 1 \\
\hline Living with partner & $0.49^{\star \star \star}(0.44-0.54)$ & $0.92(0.74-1.16)$ & $0.57^{\star \star \star}(0.43-0.75)$ & $0.29^{\star \star \star}(0.23-0.38)$ & $0.58^{*}(0.37-0.92)$ & $0.50^{\star \star \star}(0.46-0.56)$ \\
\hline Widowed & $2.16^{\star \star \star}(2.01-2.32)$ & $\begin{array}{l}1.35^{\star \star \star}(1.14- \\
1.61)\end{array}$ & $2.11^{\star \star \star}(1.82-2.45)$ & $1.73^{\star \star \star}(1.55-1.93)$ & $0.89(0.63-1.24)$ & $2.09^{\star \star \star}(1.95-2.24)$ \\
\hline Divorced/separated & $1.06(0.94-1.19)$ & $1.27(0.95-1.69)$ & $1.01(0.76-1.34)$ & $0.99(0.81-1.20)$ & $1.49(0.99-2.22)$ & $1.11(0.99-1.24)$ \\
\hline Never married & $0.22^{\star \star \star}(0.21-0.23)$ & $\begin{array}{l}0.66^{\star \star \star}(0.59- \\
0.75)\end{array}$ & $0.31^{\star \star \star}(0.27-0.35)$ & $0.19^{\star \star \star}(0.17-0.21)$ & $\begin{array}{l}0.37^{\star \star \star}(0.30- \\
0.45)\end{array}$ & $0.24^{\star \star \star}(0.23-0.26)$ \\
\hline \multicolumn{7}{|l|}{ Employment status } \\
\hline Not working & 1 & 1 & 1 & 1 & 1 & 1 \\
\hline Working & $0.74^{\star \star \star}(0.70-0.78)$ & $\begin{array}{l}0.84^{\star *}(0.74- \\
0.95)\end{array}$ & $0.56^{\star \star \star}(0.49-0.64)$ & $0.67^{\star \star \star}(0.61-0.74)$ & $0.86(0.69-1.07)$ & $0.78^{\star \star \star}(0.75-0.82)$ \\
\hline \multicolumn{7}{|l|}{ Religion } \\
\hline No religion & 1 & 1 & 1 & 1 & 1 & 1 \\
\hline Christian & $1.69^{\star * \star}(1.54-1.84)$ & $1.20(0.99-1.45)$ & $1.51^{\star \star \star}(1.21-1.87)$ & $1.38^{\star \star \star}(1.18-1.61)$ & $1.43(0.99-2.06)$ & $1.57^{\star \star \star}(1.45-1.70)$ \\
\hline Muslim & $1.35(0.95-1.91)$ & $1.44(0.72-2.88)$ & $2.85^{\star \star \star}(1.57-5.15)$ & $3.09^{\star \star \star}(2.03-4.71)$ & $2.47(0.87-7.03)$ & $1.71^{\star \star \star}(1.29-2.26)$ \\
\hline Traditional & $1.40^{\star \star \star}(1.24-1.57)$ & $0.89(0.68-1.16)$ & $0.86(0.63-1.19)$ & $1.17(0.95-1.45)$ & $0.62(0.34-1.12)$ & $1.27^{\star \star \star}(1.13-1.39)$ \\
\hline Others & $2.06^{\star \star \star}(1.67-2.56)$ & $\begin{array}{l}1.97^{\star \star \star}(1.26- \\
3.08)\end{array}$ & $3.48^{\star \star \star}(2.32-5.21)$ & $2.33^{\star \star \star}(1.67-3.27)$ & $1.71(0.71-4.10)$ & $2.14^{\star \star \star}(1.78-2.59)$ \\
\hline \multicolumn{7}{|l|}{ Education } \\
\hline No formal education & 1 & 1 & 1 & 1 & 1 & 1 \\
\hline
\end{tabular}




\begin{tabular}{|c|c|c|c|c|c|c|}
\hline Primary & $1.06(0.91-1.22)$ & $\begin{array}{l}1.57^{\star \star}(1.15- \\
2.15)\end{array}$ & $1.57^{\star \star}(1.17-2.12)$ & $1.00(0.78-1.27)$ & $1.56(0.76-3.24)$ & $1.04(0.91-1.20)$ \\
\hline Secondary & $0.72^{\star \star \star}(0.67-0.77)$ & $0.98(0.82-1.16)$ & $1.02(0.87-1.20)$ & $0.80^{\star * \star}(0.71-0.89)$ & $1.21(0.82-1.79)$ & $0.73^{\star \star \star}(0.68-0.78)$ \\
\hline Tertiary & $0.21^{\star * \star}(0.20-0.22)$ & $\begin{array}{l}0.61^{\star \star \star}(0.52- \\
0.70)\end{array}$ & $0.35^{\star \star *}(0.30-0.40)$ & $0.27^{\star \star \star}(0.25-0.30)$ & $1.12(0.80-1.56)$ & $0.24^{\star \star \star}(0.23-0.25)$ \\
\hline \multicolumn{7}{|l|}{ Ever smoked } \\
\hline No & 1 & 1 & 1 & 1 & 1 & 1 \\
\hline Yes & $1.80^{\star \star \star}(1.64-1.98)$ & $\begin{array}{l}2.06^{\star \star \star}(1.73- \\
2.45)\end{array}$ & $2.69^{\star \star \star}(2.25-3.21)$ & $1.65^{\star \star \star}(1.40-1.94)$ & $\begin{array}{l}2.94^{\star \star \star}(2.19- \\
3.95)\end{array}$ & $2.01^{\star \star \star}(1.85-2.19)$ \\
\hline \multicolumn{7}{|l|}{ Exercise } \\
\hline Never & 1 & 1 & 1 & 1 & 1 & 1 \\
\hline Rarely & $0.66^{\star \star \star}(0.60-0.73)$ & $0.88(0.71-1.09)$ & $0.85(0.68-1.06)$ & $0.81^{*}(0.69-0.96)$ & $1.30(0.92-1.85)$ & $0.74^{\star \star \star}(0.68-0.80)$ \\
\hline Once a week & $0.53^{\star \star \star}(0.50-0.57)$ & $0.89(0.77-1.01)$ & $0.88(0.77-1.01)$ & $0.65^{\star \star \star}(0.58-0.73)$ & $\begin{array}{l}1.50^{\star \star \star}(1.22- \\
1.85)\end{array}$ & $0.62^{\star \star \star}(0.58-0.65)$ \\
\hline \multicolumn{7}{|c|}{$\begin{array}{l}\text { Satisfaction level of } \\
\text { life }\end{array}$} \\
\hline Dissatisfied & 1 & 1 & 1 & 1 & 1 & 1 \\
\hline Satisfied & $0.99(0.95-1.05)$ & $0.96(0.86-1.06)$ & $0.88^{\star}(0.77-0.99)$ & $0.93(0.85-1.02)$ & $0.91(0.73-1.14)$ & $0.98(0.94-1.03)$ \\
\hline Very satisfied & $1.07^{\star}(1.01-1.14)$ & $1.04(0.91-1.19)$ & $1.02(0.88-1.19)$ & $1.08(0.96-1.21)$ & $\begin{array}{l}1.54^{\star \star \star}(1.20- \\
1.98)\end{array}$ & $1.10^{\star \star}(1.04-1.17)$ \\
\hline \multicolumn{7}{|c|}{ Survey wave years } \\
\hline 2008 & 1 & 1 & 1 & 1 & 1 & 1 \\
\hline 2011 & $0.70^{\star \star \star}(0.66-0.75)$ & $\begin{array}{l}0.78^{\star \star \star}(0.67- \\
0.90)\end{array}$ & $0.60^{\star \star \star}(0.51-0.70)$ & $0.90(0.80-1.02)$ & $0.67^{*}(0.48-0.94)$ & $0.69^{\star \star \star}(0.65-0.74)$ \\
\hline 2012 & $1.11^{\star \star \star}(1.04-1.18)$ & $1.01(0.88-1.15)$ & $0.84^{*}(0.73-0.97)$ & $1.21^{\star *}(1.08-1.35)$ & $0.89(0.65-1.22)$ & $1.04(0.98-0.74)$ \\
\hline 2015 & $0.47^{\star \star \star}(0.44-0.51)$ & $\begin{array}{l}0.60^{\star \star \star}(0.51- \\
0.70)\end{array}$ & $0.34^{\star \star \star}(0.28-0.42)$ & $0.43^{\star \star \star}(0.37-0.49)$ & $1.16(0.86-1.56)$ & $0.49^{\star \star \star}(0.46-0.52)$ \\
\hline 2017 & $0.63^{\star \star \star}(0.58-0.67)$ & $\begin{array}{l}0.68^{\star \star \star}(0.56- \\
0.79)\end{array}$ & $0.41^{\star \star \star}(0.35-0.49)$ & $0.69^{\star \star \star}(0.61-0.79)$ & $1.40^{\star}(1.06-1.87)$ & $0.63^{\star \star \star}(0.59-0.67)$ \\
\hline
\end{tabular}

Exponentiated coefficients; $95 \%$ confidence intervals in brackets

$\star p<0.05, * \star p<0.01, * \star \star p<0.001,1=$ Reference Category

Table 4 shows results of the multivariable logistic regression of the association between socio-demographic characteristics of respondents and noncommunicable diseases. Respondents aged 65+ had the highest of being diagnosed of high blood pressure [aOR=53.04, 95\% $\mathrm{Cl}=42.33-62.06], \mathrm{Asthma}$ [aOR $=2.43,95 \% \mathrm{Cl}=1.93-3.04]$, heart problem [aOR $=9.18,95 \% \mathrm{Cl}=6.93-12.15]$, diabetes [aOR $=46.26,95 \% \mathrm{Cl}=34.00-62.93]$, cancer [aOR $=5.26,95 \% \mathrm{Cl}=3.44-8.04]$ and at least one NCD [aOR $=22.15,95 \% \mathrm{Cl}=19.76-24.83]$, compared to those aged 18-24. Compared to males, females were more likely to have high blood pressure $[\mathrm{aOR}=2.03,95 \% \mathrm{Cl}=1.91-2.16]$, Asthma [aOR $=1.48,95 \% \mathrm{Cl}=1.32-1.66]$, heart problem [aOR=1.86, 95\% $\mathrm{Cl}=1.61-2.15]$, diabetes [aOR $=1.17,95 \%$ $\mathrm{Cl}=1.06-1.29]$, cancer [aOR $=1.52,95 \% \mathrm{Cl}=1.23-1.89$ ] and at least one $\mathrm{NCD}$ [aOR $=1.84,95 \% \mathrm{Cl}=1.74-1.94]$. There were higher odds of having high blood pressure $[\mathrm{aOR}=1.83,95 \% \mathrm{Cl}=1.91-2.16]$ among coloured South Africans, higher odds of Asthma [aOR $=2.92,95 \% \mathrm{Cl}=1.93-4.41]$ among Asians/Indians, higher odds of heart problem among White South Africans [AOR=1.81, 95\% Cl=1.44-2.28], higher odds of cancer [aOR $=2.77,95 \% \mathrm{Cl}=1.98-3.89$ ] among Asians/Indians, higher odds of diabetes among White South Africans [aOR =3.92, 95\% Cl=2.91-5.29] and higher odds of at least one NCD [aOR $=1.75,95 \%$ $\mathrm{Cl}=1.63-1.88]$ among coloured South Africans, compared to African South Africans.

Respondents who were living with a partner $[\mathrm{aOR}=1.31,95 \% \mathrm{Cl}=1.04-1.66]$ and those who were never married [aOR $=1.30,95 \% \mathrm{Cl}=1.13-1.48]$ were $\mathrm{more}$ likely to have asthma, compared to those who were married. Over the ten year period, respondents who were Christians, were more likely to have high blood pressure [aOR $=1.22,95 \% \mathrm{Cl}=1.10-1.35]$ and at least one $\mathrm{NCD}[\mathrm{aOR}=1.18,95 \% \mathrm{Cl}=1.08-1.29]$, compared to those who had no religion. Whiles respondents who had primary education had the highest odds of having high blood pressure [aOR $=1.42,95 \% \mathrm{Cl}=1.21-1.67], \mathrm{Asthma}$ [aOR $=1.64,95 \% \mathrm{Cl}=1.22-2.21]$, heart problem [aOR $=1.91,95 \% \mathrm{Cl}=1.41-2.59]$ and at least one $\mathrm{NCD}$ [aOR $=1.38,95 \% \mathrm{Cl}=1.18-1.62]$ compared to those with no formal education, those with tertiary education had the highest likelihood of having cancer [aOR $=1.38,95 \% \mathrm{Cl}=1.20-1.57]$ and diabetic $[\mathrm{aOR}=1.76,95 \% \mathrm{Cl}=1.18-2.61]$.

There were higher odds of having high blood pressure [aOR $=1.33,95 \% \mathrm{Cl}=1.19-1.49]$, Asthma [aOR $=1.74,95 \% \mathrm{Cl}=1.44-2.11]$, heart problem [aOR $=2.01,95 \%$ $\mathrm{Cl}=1.65-2.45]$, cancer [aOR $=1.72,95 \% \mathrm{Cl}=1.24-2.39]$ and at least one $\mathrm{NCD}$ [aOR $=1.49,95 \% \mathrm{Cl}=1.35-1.65]$ among respondents who smoked, compared to nonsmokers. The odds of having heart problem $[\mathrm{aOR}=1.41,95 \% \mathrm{Cl}=1.20-1.65]$ and cancer $[\mathrm{aOR}=1.38,95 \% \mathrm{Cl}=1.08-1.76]$ were higher among those who exercised 
once a week, compared to those who never exercised. Respondents who very satisfied with life were less likely to have heart problem $[\mathrm{aOR}=0.83,95 \% \mathrm{Cl}=0.70$ 0.98 ] while those who were satisfied with life were less likely to have cancer [aOR $=0.71,95 \% \mathrm{Cl}=0.56-0.90$ ], compared to those who were dissatisfied with life.

In terms of survey years, high prevalence of high blood pressure [aOR $=1.25,95 \% \mathrm{Cl}=1.16-1.34]$, diabetes [aOR=1.31, 95\% $\mathrm{Cl}=1.16-1.48$ ] and at least one $\mathrm{NCD}$ [aOR=1.18, 95\% Cl=1.10-1.26], were recorded in 2012, compared to 2008. On the other hand, respondent who were working were less likely to have high blood pressure [aOR=0.82, 95\% $\mathrm{Cl}=0.77-0.88]$, heart problem [aOR=1.68, 95\% $\mathrm{Cl}=0.58-0.79]$, diabetes [aOR=0.77, 95\% $\mathrm{Cl}=0.69-0.85]$, cancer [aOR=0.78, 95\% $\mathrm{Cl}=0.61$ $0.99]$ and at least one $\mathrm{NCD}[\mathrm{aOR}=0.86,95 \% \mathrm{Cl}=0.81-0.91]$, compared to those who were not working.

Table 4: Multivariable logistic regression of the association between socio-demographic, behavioural characteristics and non-communicable diseases 


\begin{tabular}{|c|c|c|c|c|c|c|}
\hline Variables & High blood pressure & Asthma & Heart problem & Diabetes & Cancer & At least one NCD \\
\hline \multicolumn{7}{|l|}{$\mathrm{N}=64,735$} \\
\hline & AOR $(95 \% \mathrm{Cl})$ & AOR $(95 \% \mathrm{Cl})$ & AOR (95\% Cl) & AOR $(95 \% \mathrm{Cl})$ & AOR $(95 \% \mathrm{Cl})$ & AOR $(95 \% \mathrm{Cl})$ \\
\hline \multicolumn{7}{|l|}{ Age } \\
\hline $18-24$ & 1 & 1 & 1 & 1 & 1 & 1 \\
\hline $25-34$ & $3.13^{\star \star \star}(2.70-3.64)$ & $1.06(0.71-1.24)$ & $1.29(0.98-1.69)$ & $2.03^{\star \star \star}(1.45-2.82)$ & $1.02(0.70-1.48)$ & $1.72^{\star \star \star}(1.56-1.89)$ \\
\hline $35-44$ & $8.41^{\star \star *}(7.27-9.73)$ & $\begin{array}{l}1.41^{* \star *}(1.19- \\
1.68)\end{array}$ & $2.34^{\star \star \star}(1.79-3.06)$ & $8.66^{\star \star \star}(6.42-11.67)$ & $\begin{array}{l}1.80^{\star \star \star}(1.21- \\
2.68)\end{array}$ & $3.92^{\star \star *}(3.56-4.31)$ \\
\hline $45-54$ & $\begin{array}{l}21.66^{* \star}(18.68- \\
25.12)\end{array}$ & $\begin{array}{l}2.29^{\star \star \star} \\
2.76)\end{array}$ & $5.05^{\star \star \star}(3.86-6.61)$ & $\begin{array}{l}23.42^{\star \star \star}(17.38- \\
31.57)\end{array}$ & $\begin{array}{l}3.31^{\star \star \star}(2.24- \\
4.88)\end{array}$ & $9.32^{\star \star \star}(8.42-10.30)$ \\
\hline $55-64$ & $\begin{array}{l}41.48^{* \star \star}(35.63- \\
48.29)\end{array}$ & $\begin{array}{l}2.42^{\star \star \star}(2.00- \\
3.04)\end{array}$ & $\begin{array}{l}7.71^{* \star *}(5.86- \\
10.15)\end{array}$ & $\begin{array}{l}45.63^{\star \star \star}(33.81- \\
61.58)\end{array}$ & $\begin{array}{l}3.65^{\star \star \star}(2.42- \\
5.50)\end{array}$ & $\begin{array}{l}17.32^{\star \star \star}(15.57- \\
19.28)\end{array}$ \\
\hline $65+$ & $\begin{array}{l}53.04^{\star \star \star}(42.33- \\
62.06)\end{array}$ & $\begin{array}{l}2.43^{\star \star \star}(1.93- \\
3.04)\end{array}$ & $\begin{array}{l}9.18^{\star \star \star}(6.93- \\
12.15)\end{array}$ & $\begin{array}{l}46.26^{* \star \star}(34.00- \\
62.93)\end{array}$ & $\begin{array}{l}5.26^{\star \star \star}(3.44- \\
8.04)\end{array}$ & $\begin{array}{l}22.15^{\star \star \star}(19.76- \\
24.83)\end{array}$ \\
\hline \multicolumn{7}{|l|}{ Gender } \\
\hline Male & 1 & 1 & 1 & 1 & 1 & 1 \\
\hline Female & $2.03^{\star \star \star}(1.91-2.16)$ & $\begin{array}{l}1.48^{\star \star \star}(1.32- \\
1.66)\end{array}$ & $1.86^{\star \star \star}(1.61-2.15)$ & $1.17^{\star *}(1.06-1.29)$ & $\begin{array}{l}1.52^{\star \star \star}(1.23- \\
1.89)\end{array}$ & $1.84^{\star \star \star}(1.74-1.94)$ \\
\hline \multicolumn{7}{|l|}{ Population group } \\
\hline African & 1 & 1 & 1 & 1 & 1 & 1 \\
\hline Coloured & $1.83^{\star \star \star}(1.91-2.16)$ & $\begin{array}{l}1.43^{\star * \star}(1.24- \\
1.67)\end{array}$ & $1.51^{\star \star \star}(1.37-1.79)$ & $1.58^{\star \star \star}(1.39-1.79)$ & $1.17(0.84-1.63)$ & $1.75^{\star \star *}(1.63-1.88)$ \\
\hline Asian/Indian & $1.32^{*}(1.01-1.73)$ & $\begin{array}{l}2.92^{\star \star \star} \\
4.41)\end{array}$ & $1.79^{*}(1.23-2.85)$ & $2.77^{\star \star \star}(1.98-3.89)$ & $0.86(0.32-2.28)$ & $1.48^{\star \star \star}(1.17-1.89)$ \\
\hline White & $0.86^{*}(0.76-0.98)$ & $\begin{array}{l}2.26^{* * *}(1.83- \\
2.80)\end{array}$ & $1.81^{\star \star \star}(1.44-2.28)$ & $0.81^{\star}(0.66-0.99)$ & $\begin{array}{l}3.92^{\star \star \star}(2.91- \\
5.29)\end{array}$ & $1.21^{\star \star \star}(1.08-1.36)$ \\
\hline \multicolumn{7}{|l|}{ Marital status } \\
\hline Married & 1 & 1 & 1 & 1 & 1 & 1 \\
\hline Living with partner & $1.00(0.88-1.13)$ & $1.31^{*}(1.04-1.66)$ & $1.00(0.75-1.32)$ & $0.61^{\star \star \star}(0.47-0.78)$ & $1.53(0.94-2.49)$ & $1.01(0.90-1.12)$ \\
\hline Widowed & $0.97(0.89-1.05)$ & $1.05(0.87-1.26)$ & $1.16(0.75-1.32)$ & $1.01(0.90-1.15)$ & $0.93(0.64-1.36)$ & $1.00(0.92-1.08)$ \\
\hline Divorced/separated & $0.88^{\star}(0.77-1.00)$ & $1.22(1.13-1.48)$ & $0.88(0.65-1.18)$ & $0.88(0.72-1.08)$ & $1.24(0.82-1.89)$ & $0.94(0.83-1.06)$ \\
\hline Never married & $0.82^{\star \star \star}(0.77-0.88)$ & $\begin{array}{l}1.30^{\star \star \star}(1.13- \\
1.48)\end{array}$ & $0.94(0.80-1.10)$ & $0.78^{\star \star \star}(0.70-0.88)$ & $0.90(0.68-1.19)$ & $0.84^{\star \star \star}(0.79-0.90)$ \\
\hline \multicolumn{7}{|l|}{ Employment status } \\
\hline Not working & 1 & 1 & 1 & 1 & 1 & 1 \\
\hline Working & $0.85^{\star \star \star}(0.79-0.90)$ & $0.90(0.80-1.01)$ & $0.68^{\star \star \star}(0.58-0.79)$ & $0.77^{\star \star \star}(0.69-0.85)$ & $0.78^{*}(0.61-0.99)$ & $0.86^{\star * \star}(0.81-0.91)$ \\
\hline \multicolumn{7}{|l|}{ Religion } \\
\hline No religion & 1 & 1 & 1 & 1 & 1 & 1 \\
\hline Christian & $1.22^{\star \star \star}(1.10-1.35)$ & $1.09(0.91-1.30)$ & $1.12(0.90-1.40)$ & $1.04(0.88-1.23)$ & $1.10(0.75-1.61)$ & $1.18^{\star \star \star}(1.08-1.29)$ \\
\hline Muslim & $0.69(0.46-1.04)$ & $0.86(0.42-1.76)$ & $1.48(0.75-2.92)$ & $1.11(0.66-1.87)$ & $2.13(0.69-6.56)$ & $0.95(0.66-1.36)$ \\
\hline Traditional & $1.05(0.92-1.20)$ & $0.84(0.65-1.09)$ & $0.74(0.53-1.02)$ & $0.94(0.76-1.17)$ & $0.65(0.36-1.18)$ & $0.99(0.88-1.11)$ \\
\hline Others & $1.08(0.80-1.46)$ & $0.90(0.56-1.46)$ & $1.67(0.99-2.81)$ & $0.69(0.45-1.06)$ & $1.24(0.45-3.37)$ & $1.14(0.88-1.48)$ \\
\hline \multicolumn{7}{|l|}{ Education } \\
\hline No formal education & 1 & 1 & 1 & 1 & 1 & 1 \\
\hline Primary & $1.42^{\star \star \star}(1.21-1.67)$ & $1.64^{\star \star}(1.22-2.21)$ & $1.91^{\star \star \star}(1.41-2.59)$ & $1.24(0.97-1.59)$ & $1.50(0.69-3.24)$ & $1.38^{\star \star \star}(1.18-1.62)$ \\
\hline Secondary & $1.22^{\star \star *}(1.13-1.32)$ & $1.06(0.90-1.25)$ & $1.50^{\star \star \star}(1.41-2.59)$ & $1.22^{\star \star \star}(1.08-1.38)$ & $1.49^{*}(1.00-2.23)$ & $1.22^{\star \star \star}(1.13-1.31)$ \\
\hline
\end{tabular}




\begin{tabular}{|c|c|c|c|c|c|c|}
\hline Tertiary & $1.12^{\star \star \star}(1.03-1.21)$ & 0.91(0.77-1.09) & $1.14(0.94-1.39)$ & $1.38^{\star \star \star}(1.20-1.57)$ & $\begin{array}{l}1.76^{\star *}(1.18- \\
2.61)\end{array}$ & $1.08(1.00-1.16)$ \\
\hline \multicolumn{7}{|l|}{ Ever smoked } \\
\hline No & 1 & 1 & 1 & 1 & 1 & 1 \\
\hline Yes & $1.33^{\star \star \star}(1.19-1.49)$ & $\begin{array}{l}1.74^{\star \star \star}(1.44- \\
2.11)\end{array}$ & $2.01^{\star \star \star}(1.65-2.45)$ & $1.03(0.87-1.23)$ & $\begin{array}{l}1.72^{\star \star}(1.24- \\
2.39)\end{array}$ & $1.49^{\star \star \star}(1.35-1.65)$ \\
\hline \multicolumn{7}{|l|}{ Exercise } \\
\hline Never & 1 & 1 & 1 & 1 & 1 & 1 \\
\hline Rarely & $0.95(0.85-1.06)$ & $1.04(0.85-1.26)$ & $1.15(0.91-1.45)$ & $1.09(0.72-1.31)$ & $1.26(0.87-1.81)$ & $1.02(0.93-1.12)$ \\
\hline Once a week & $1.01(0.93-1.09)$ & $1.10(0.97-1.25)$ & $1.41^{\star \star \star}(1.20-1.65)$ & $1.13(0.92-1.31)$ & $1.38^{\star}(1.08-1.76)$ & $1.07(1.00-1.15)$ \\
\hline \multicolumn{7}{|c|}{$\begin{array}{l}\text { Satisfaction level of } \\
\text { life }\end{array}$} \\
\hline Dissatisfied & 1 & 1 & 1 & 1 & 1 & 1 \\
\hline Satisfied & $1.02(0.96-1.08)$ & $0.95(0.78-1.05)$ & $0.87^{*}(0.77-0.99)$ & $0.94(0.86-1.04)$ & $\begin{array}{l}0.71^{* \star}(0.56- \\
0.90)\end{array}$ & $0.99(0.94-1.04)$ \\
\hline Very satisfied & $1.03(0.95-1.11)$ & $0.91(0.78-1.05)$ & $0.83^{\star}(0.70-0.98)$ & $0.97(0.85-1.10)$ & $0.81(0.61-1.09)$ & $1.01(0.95-1.09)$ \\
\hline \multicolumn{7}{|c|}{ Survey wave years } \\
\hline 2008 & 1 & 1 & 1 & 1 & 1 & 1 \\
\hline 2011 & $0.73^{\star \star \star}(0.68-0.79)$ & $0.85^{*}(0.74-0.99)$ & $0.70^{\star \star \star}(0.59-0.82)$ & $0.97(0.85-1.10)$ & $0.82(0.59-1.16)$ & $0.72^{\star \star \star}(0.67-0.77)$ \\
\hline 2012 & $1.25^{\star \star \star}(1.16-1.34)$ & $1.10(0.96-1.26)$ & $0.96(0.82-1.11)$ & $1.31^{\star \star \star}(1.16-1.48)$ & $1.07(0.79-1.47)$ & $1.18^{\star \star \star}(1.10-1.26)$ \\
\hline 2015 & $0.89^{*}(0.81-0.98)$ & $0.77^{\star \star}(0.65-0.91)$ & $0.62^{\star \star \star}(0.51-0.77)$ & $0.78^{\star \star}(0.67-0.92)$ & $\begin{array}{l}1.84^{\star \star \star}(1.34- \\
2.52)\end{array}$ & $0.86^{\star \star \star}(0.80-0.93)$ \\
\hline 2017 & $0.95(0.87-1.03)$ & $0.79^{\star \star}(0.68-0.92)$ & $0.60^{\star \star \star}(0.50-0.72)$ & $0.99(0.86-1.13)$ & $\begin{array}{l}1.77^{\star \star \star}(1.32- \\
2.38)\end{array}$ & $0.91^{*}(0.85-0.98)$ \\
\hline Pseudo $\mathrm{R}^{2}$ & 0.249 & 0.035 & 0.122 & 0.185 & 0.085 & 0.215 \\
\hline $\mathrm{N}$ & 64735 & 64735 & 64735 & 64735 & 64735 & 64735 \\
\hline
\end{tabular}

Exponentiated coefficients; $95 \%$ confidence intervals in brackets

${ }^{*} p<0.05,{ }^{* \star} p<0.01, * \star * p<0.001,1=$ Reference Category

\section{Discussion}

This study, to the best of our knowledge, provides the first report of the prevalence and factors associated with the occurrence of major NCDs among the adult South African population. We found out that the occurrence of high blood pressure among the adult population in South Africa is of major concern as compared with diabetes, asthma, heart problems and cancers with age, gender, marital status, religion, level of satisfaction, education, and exercise all playing major roles in the reporting of these NCDs. It's also important to know that high blood pressure is regarded as the world's most prevalent NCD affecting billions of persons, including Africans [33, 34].

This study identified respondents age to be associated with blood pressure, heart problem, diabetes, cancer and asthma. This was observed more in adults aged $65+$ than in other age groups. Similarly, some previous reports across the world have indicated a positive relationship between age and most NCDs, especially high blood pressure [34-38]. In old adults, due to ageing, major blood vessels in the body come constricted, leading to high blood pressure (39), possibly resulting from the cumulative effects of the adults' poor behavioural habits [39]. These suggest that more priority should be paid to the treatment of NCDs in adults.

The influence of gender in the occurrence of high blood pressure, asthma, heart problem, diabetes, and cancer was highlighted in this study, similarly, as observed by Esmailnasab et al. [37]. Females of the 10 years were more likely to carry these NCDs. This is in contrast to studies by Gao et al. [36] and Arab et al. [40] who reported high blood pressure and other NCDs had been reported to occur more in men than women. This observation could be attributed to biological sex difference [41], an insufficient number of females or the disproportionate number of gender type recruited in this study. This needs to be explored further to determine why more women in SA are at higher odds of having NCDs. In contrast to the findings of Adhikari et al., [42] women in SA tend to get engaged in behaviours that increase the occurrence of NCDs. 
Widowed respondents, were more likely to have high blood pressure, asthma, heart problem, and diabetes when compared to those who were married. This result is unexpected as these widows could have been exposed to psychological, psychosocial, emotional, and physical trauma or stress. These are associated with several cardiovascular symptoms in humans, including cardiac death [43-45].

We found that even though most respondents were associated with a religion, is unprotective from having high blood pressure, asthma, diabetes, and heart problem compared to those who had no religion. This is contrary to the findings of Meng et al. [46], who suggested that religion benefits control and prevention of blood pressure in patients. The scenario is similar to other cardiovascular diseases and NCDs [45]. Religion and spiritual coping decreased the risk of hypertension among African American women, even when they are exposed to stress [47].

This study showed that smokers were at higher odds of having blood pressure, asthma, heart problem, diabetes, and cancer than non-smokers. This observation is consistent with previous findings $[41,45]$. Lack of behavioural risk factors such as smoking could serve as a protective effect against most NCDs [41].

Exercise is judged to be very protective as a sedentary lifestyle is regarded as a risk factor for many cardiovascular diseases [48]. Regular exercise is regarded as an important activity for preventing high blood pressure [49,50] and for improving life expectancy [51]. Improvement in the quality of life of patients with heart disease who participated in regular exercise has also been confirmed in a study [48]. In this study, respondents who exercised once a week were less likely to have high blood pressure, cancer and at least one NCD compared to those who never exercised. However, other studies found an inverse relationship between exercise and the risk of high blood pressure [41,52]. Regular exercise benefits the health, lower high blood pressure and reduces the risk of cardiovascular diseases. Thus, increasing physical activity levels should be a major goal at all levels of health care [53].

Our study noted that lower odds of high blood pressure, asthma, heart problem, diabetes were found among respondents with tertiary education, compared to those with no formal education. This finding is corroborated with a study that concluded that education was significantly associated with the occurrence of NCDs $[54,55]$. Also, respondents who are very satisfied with life were more likely to have high blood pressure and cancer than those who were dissatisfied with life. Higher socio-economic status is regarded as a risk factor for some cardiovascular diseases, especially hypertension [35, 36, 56, 57]. It is believed that those who are satisfied with life have more purchasing power for feast conveniently with little participation in exercise and this lifestyle easily predisposes them to NCDs [41].

\section{Strength And Limitations}

The study's major strengths were the large study participants, representativeness of the country and these surveys were conducted by well-trained indigenous enumerators who were able to capture the dataset accurately and timely. Additionally, the study gave specific and overall insights on NCDs prevalence and associated factors in SA for the 10 years. These results could be used in developing major behavioural interventions toward reducing NCDs prevalence with special consideration of the associated factors. The study's limitations were that this study did not incorporate the qualitative data. Also, the study's crosssectional nature makes it difficult to ascertain the true cause and effect relationship between NCDs and associated factors.

\section{Conclusions And Policy Implications}

This study utilized prevailing associated factors based on the literature gap about non-communicable diseases prevalence in South Africa for the period of 10year. The study concluded that among the NCDs, high blood pressure and diabetes were the prevailing diseases with the highest prevalence across the 10-year while cancer has the lowest prevalence. Socio-demographic factors associated with a high prevalence of NCDs were respondents older than 65 , those who were females, widowed and those without education or primary education whilst the behavioural factors were respondents who were smokers, those who engage in less physical activities or exercises and those who reported having been satisfied with life. South Africa's department of health and other health agencies need to strengthen existing policies and develop new interventional frameworks that will deliberately put into consideration various factors contributing to the high prevalence of NCDs identified in this study. This will not only reduce the NCDs prevalence in the country but will also reduce the morbidity and mortality levels.

\section{Abbreviations}

NCDs: Non-communicable diseases; AOR: Adjusted Odds Ratio; Cl: Confidence Interval; UOR: Unadjusted Odds Ratio; NIDS: National Income Dynamic Survey; SA: South Africa; UN: United Nations; WHO: World Health Organization; DALYs: Disability-adjusted life years; LMICs: Low-and-Middle Income Countries; DPME: Department of Planning, Monitoring and Evaluation and SALDRU: Southern Africa Labour and Development Research Unit.

\section{Declarations}

\section{Acknowledgments}

We wish to thank Department of Planning, Monitoring and Evaluation (DPME) and Southern Africa Labour and Development Research Unit (SALDRU) for providing us free access to the datasets.

\section{Authors' contributions}

OAB conceived the study. BOA, OAB, OSO, EB and AS developed the do-files and performed the analysis. OAB, BOA, IAO, AIA, and SAS developed first draft of the manuscript. $O A B$ and $B O A$ revised the manuscript and all authors read and approved the final manuscript.

Page $14 / 19$ 


\section{Authors' information}

Discipline of Public Health Medicine, School of Nursing and Public Health, University of

KwaZulu-Natal, Durban, South Africa (OAB), Department of Demography and Social Statistics, Obafemi Awolowo University, Nigeria (OAB, OSO), School of Public Health, Faculty of Health, University of Technology Sydney, Sydney, Australia (BOA), Department of Population and Health, University of Cape Coast, Cape Coast, Ghana (EB, AS), College of Public Health, Medical and Veterinary Services, James Cook University, Australia (AS), Department of Veterinary Public Health and Preventive Medicine, Faculty of Veterinary Medicine, University of Ilorin, Kwara State, Nigeria (IOA), Department of Veterinary Services, Kwara State Ministry of Agriculture, llorin, Nigeria (AIA), Discipline of Population studies, School of Built Environment and Development Studies, University of Kwazulu Natal, South Africa (SAS).

\section{Funding}

The study did not receive any funding.

\section{Availability of data and materials}

The dataset is freely available at http://www.nids.uct.ac.za/

\section{Ethics approval and consent to participate}

The Department of Planning, Monitoring and Evaluation (DPME) and Southern Africa Labour and Development Research Unit (SALDRU) obtain ethical clearance from the University of Cape Town, South Africa. During each of the surveys, either written or verbal consent was provided by the respondents. Since the data was not collected by any authors of this paper, we sought permission from National Income Dynamic Survey website and access to the data was provided after our intent for the request was assessed and approved on May 2020.

\section{Consent for publication}

Not applicable.

\section{Competing interests}

The authors declare no competing interests in the conduct of this research.

\section{References}

[1] U. Intarakamhang, A. Macaskill, and P. Prasittichok, "Mindfulness interventions reduce blood pressure in patients with non-communicable diseases: A systematic review and meta-analysis," Heliyon, vol. 6, no. 4, p. e03834, 2020/04/01/ 2020, doi: https://doi.org/10.1016/j.heliyon.2020.e03834.

[2] A. Alwan, Global status report on noncommunicable diseases 2010. World Health Organization, 2011.

[3] World Health Organization, Global status report on noncommunicable diseases 2014 (no. WHO/NMH/NVI/15.1). World Health Organization, 2014.

[4] H. N. Gouda et al., "Burden of non-communicable diseases in sub-Saharan Africa, 1990-2017: results from the Global Burden of Disease Study 2017," The Lancet Global Health, vol. 7, no. 10, pp. e1375-e1387, 2019/10/01/ 2019, doi: https://doi.org/10.1016/S2214-109X(19)30374-2.

[5] S. Rath, P. Yu, and S. Srinivas, "Challenges of non-communicable diseases and sustainable development of China and India," Acta Ecologica Sinica, vol. 38, no. 2, pp. 117-125, 2018/04/01/ 2018, doi: https://doi.org/10.1016/j.chnaes.2017.06.012.

[6] S. K. Sharma, D. Vishwakarma, and P. Puri, "Gender disparities in the burden of non-communicable diseases in India: Evidence from the cross-sectional study," Clinical Epidemiology and Global Health, vol. 8, no. 2, pp. 544-549, 2020/06/01/ 2020, doi: https://doi.org/10.1016/j.cegh.2019.11.011.

[7] D. J. Hunter and K. S. Reddy, "Noncommunicable diseases," New England Journal of Medicine, vol. 369, no. 14, pp. 1336-1343, 2013. [Online]. Available: https://www.nejm.org/doi/pdf/10.1056/NEJMra1109345?articleTools=true.

[8] World Health Organization, "65th World Health Assembly closes with new global health measures [Internet]. Geneva: WHO; $2012, "$ ed, 2012.

[9] K. V. Narayan, M. K. Ali, and J. P. Koplan, "Global noncommunicable diseases-where worlds meet," New England Journal of Medicine, vol. 363, no. 13, pp. 1196-1198, 2010. [Online]. Available: https://www.nejm.org/doi/pdf/10.1056/NEJMp1002024?articleTools=true.

[10] K. Hofman, "Guest editorial: non-communicable diseases in South Africa: a challenge to economic development," South African Medical Journal, vol. 104, no. 10 , pp. 647-647, 2014.

[11] R. Uddin, E.-Y. Lee, S. R. Khan, M. S. Tremblay, and A. Khan, "Clustering of lifestyle risk factors for non-communicable diseases in 304,779 adolescents from 89 countries: A global perspective," Preventive Medicine, vol. 131, p. 105955, 2020/02/01/ 2020, doi: https://doi.org/10.1016/j.ypmed.2019.105955.

[12] M. Spires, P. Delobelle, D. Sanders, T. Puoane, P. Hoelzel, and R. Swart, "Diet-related non-communicable diseases in South Africa: determinants and policy responses," South African Health Review, vol. 2016, no. 1, pp. 35-42, 2016. 
[13] M. Ezzati and E. Riboli, "Behavioral and dietary risk factors for noncommunicable diseases," New England Journal of Medicine, vol. 369, no. 10, pp. 954964, 2013. [Online]. Available: https://www.nejm.org/doi/pdf/10.1056/NEJMra1203528?articleTools=true.

[14] S. Yaya, M. Ekholuenetale, and G. Bishwajit, "Differentials in prevalence and correlates of metabolic risk factors of non-communicable diseases among women in sub-Saharan Africa: evidence from 33 countries," BMC Public Health, vol. 18, no. 1, p. 1168, 2018. [Online]. Available:

https://bmcpublichealth.biomedcentral.com/track/pdf/10.1186/s12889-018-6085-2.

[15] N. Curbing, "Noncommunicable diseases in africa: youth are key to curbing the epidemic and achieving sustainable development," Washington: Population Reference Bureau, 2015.

[16] B. M. Mayosi, A. J. Flisher, U. G. Lalloo, F. Sitas, S. M. Tollman, and D. Bradshaw, "The burden of non-communicable diseases in South Africa," The lancet, vol. 374, no. 9693, pp. 934-947, 2009. [Online]. Available: https://www.thelancet.com/journals/lancet/article/PIIS0140-6736(09)61087-4/fulltext.

[17] B. Nojilana et al., "Persistent burden from non-communicable diseases in South Africa needs strong action," South African Medical Journal, vol. 106, no. 5, pp. 436-437, 2016.

[18] T. R. Puoane, L. P. Tsolekile, S. Caldbick, E. U. Igumbor, K. Meghnath, and D. Sanders, "Chronic non-communicable diseases in South Africa: Progress and challenges: Social and environmental determinants of health," South African Health Review, vol. 2012, no. 1, pp. 115-126, 2012.

[19] T. Oni, E. Youngblood, A. Boulle, N. McGrath, R. J. Wilkinson, and N. S. Levitt, "Patterns of HIV, TB, and non-communicable disease multi-morbidity in periurban South Africa-a cross sectional study," BMC infectious diseases, vol. 15, no. 1, pp. 1-8, 2015. [Online]. Available:

https://bmcinfectdis.biomedcentral.com/track/pdf/10.1186/s12879-014-0722-x.

[20] F. X. Gomez-Olive, M. Thorogood, B. Clark, K. Kahn, and S. Tollman, "Self-reported health and health care use in an ageing population in the Agincourt subdistrict of rural South Africa," Global health action, vol. 6, no. 1, p. 19305, 2013.

[21] S. Dalal et al., "Non-communicable diseases in sub-Saharan Africa: what we know now," International journal of epidemiology, vol. 40, no. 4, pp. 885-901, 2011. [Online]. Available: https://watermark.silverchair.com/dyr050.pdf?

token=AQECAHi208BE490oan9kkhW_Ercy7Dm3ZL_9Cf3qfKAc485ysgAAAqAwggKcBgkqhkiG9w0BBwagggKNMIICiQIBADCCAoIGCSqGSIb3DQEHATAeBglghk q0qlsk5YrarKh6c9sxy5iXHvTpOAqkGaLmuqdqFH0693TzS09vVc3Jdv4DbYsQnizBSbEZco6z6ReBUckVysyBLZuuJuQCe9KAYHxxRBsLiNrJY_FQTnz2KxKkyWI TuC49xpC7ow6KVNNZNG-8qdgL67pi7tSzoSpn6RMxxsq41cCCattHn50kPXdL9v8iSOKfVE4yrNP1as5Pf-

GJL6x4fSM23x4Sw9gdlzYUaLc8YC7eQu8hza91SEo7CWaTD85TUwYZasAPja9zV7dfKLq11luOoWwOb0WMEeL3Ztbc1a9suCMPnoK2sApl8txFU_D_o1gWI50pLvFwoHOEfnEw5HqotOYNF3FcNTP65Fq3ij4vr0w5eyc4e4NxW9_rntNdZcF29bvSKZLdc5WmwVdX9dZKQhX3MKinkDbfZ3maPaaaT6BumJ8EitDTd! d-U5Wod4kdV1QgcZED4nmycn7aOc35ZgPG1aHJ1URAYxT7_aqIDVw-fUw-haYwBIJn9HdTWTFio0b9E-

NsuNPqIYoF4vONieytVBT_zAe1erTB5WucIMKbyPu2NFnavxxM9SXe9cNFgnPkgVTkXMaqgBPSLpXBMioqdnEEBtprTa1ra7z83S9_hi4xtqqPe4DPfMxsuGi2nB5

[22] N. Solomons, H. S. Kruger, and T. R. Puoane, "Adherence challenges encountered in an intervention programme to combat chronic non-communicable diseases in an urban black community, Cape Town," Health SA Gesondheid, vol. 22, pp. 70-78, 2017/12/01/ 2017, doi:

https://doi.org/10.1016/j.hsag.2016.11.006.

[23] ECA, "Demographic Profile of African Countries," United Nations Economic Commission for West Africa Economic Commission for Africa, 2016. [Online]. Available: https://www.uneca.org/sites/default/files/PublicationFiles/demographic_profile_rev_april_25.pdf

[24] R. Norman, D. Bradshaw, M. Schneider, D. Pieterse, and P. Groenewald, "Revised burden of disease estimates for the comparative risk factor assessment, South Africa 2000," Cape Town: Medical Research Council, 2006.

[25] NDH, "Strategic plan for the prevention and control of non-communicable diseases 2013-17," ed: National Department of Health Pretoria, 2013.

[26] N. Phaswana-Mafuya, K. Peltzer, W. Chirinda, A. Musekiwa, and Z. Kose, "Sociodemographic predictors of multiple non-communicable disease risk factors among older adults in South Africa," Global health action, vol. 6, no. 1, p. 20680, 2013.

[27] South Africa Department of Health, "NSDA: A Long and Healthy Life for All South Africans," ed: Department of Health Pretoria, 2013.

[28] M. Leibbrandt, I. Woolard, and L. de Villiers, "Methodology: Report on NIDS wave 1," Technical paper, vol. 1, 2009.

[29] L. Allen et al., "Socioeconomic status and non-communicable disease behavioural risk factors in low-income and lower-middle-income countries: a systematic review," The Lancet Global Health, vol. 5, no. 3, pp. e277-e289, 2017.

[30] V. Kontis et al., "Contribution of six risk factors to achieving the $25 \times 25$ non-communicable disease mortality reduction target: a modelling study," The Lancet, vol. 384, no. 9941, pp. 427-437, 2014.

[31] B. Bista, R. R. Dhungana, B. Chalise, and A. R. Pandey, "Prevalence and determinants of non-communicable diseases risk factors among reproductive aged women of Nepal: Results from Nepal Demographic Health Survey 2016," PloS one, vol. 15, no. 3, p. e0218840, 2020.

[32] J. L. Castilho et al., "Trends and predictors of non-communicable disease multimorbidity among adults living with HIV and receiving antiretroviral therapy in Brazil," Journal of the International AIDS Society, vol. 22, no. 1, p. e25233, 2019. 
[33] T. Lawoyin et al., "Prevalence of cardiovascular risk factors in an African, urban inner city community," West African journal of medicine, vol. 21, no. 3, pp. 208-211, 2002.

[34] F. Princewel et al., "Prevalence and risk factors associated with hypertension among adults in a rural setting: the case of Ombe, Cameroon," The Pan African Medical Journal, vol. 34, 2019.

[35] J. F. Wamala, Z. Karyabakabo, D. Ndungutse, and D. Guwatudde, "Prevalence factors associated with hypertension in Rukungiri district, Uganda-a community-based study," African health sciences, vol. 9, no. 3, 2009.

[36] Y. Gao et al., "Prevalence of hypertension in China: a cross-sectional study," PloS one, vol. 8, no. 6, p. e65938, 2013.

[37] N. Esmailnasab, G. Moradi, and A. Delaveri, "Risk factors of non-communicable diseases and metabolic syndrome," Iranian journal of public health, vol. 41, no. 7, p. 77, 2012.

[38] K. Thankappan, G. Mini, M. Daivadanam, G. Vijayakumar, P. Sarma, and M. Nichter, "Smoking cessation among diabetes patients: results of a pilot randomized controlled trial in Kerala, India," BMC Public Health, vol. 13, no. 1, p. 47, 2013.

[39] F. Wu et al., "Common risk factors for chronic non-communicable diseases among older adults in China, Ghana, Mexico, India, Russia and South Africa: the study on global AGEing and adult health (SAGE) wave 1," BMC public health, vol. 15, no. 1, pp. 1-13, 2015.

[40] A. Arab et al., "The effect of a lifestyle modification education on adiposity measures in overweight and obese nonalcoholic fatty liver disease patients," International journal of preventive medicine, vol. 8, 2017.

[41] L. Yun, P. J. Chen, A. Singh, A. F. Agrawal, and H. D. Rundle, "The physical environment mediates male harm and its effect on selection in females," Proceedings of the Royal Society B: Biological Sciences, vol. 284, no. 1858, p. 20170424, 2017.

[42] K. Adhikari, N. Gupta, and A. Koshy, "Gender Differences on Risk Factors of Noncommunicable Diseases â€"A Community Based Cross-sectional Study in Central Nepal," Journal of Nepal Health Research Council, 2014.

[43] M. M. Burg et al., "Low perceived social support and post-myocardial infarction prognosis in the enhancing recovery in coronary heart disease clinical trial: the effects of treatment," Psychosomatic medicine, vol. 67, no. 6, pp. 879-888, 2005.

[44] R. C. Ziegelstein, "Acute emotional stress and cardiac arrhythmias," Jama, vol. 298, no. 3, pp. 324-329, 2007.

[45] F. A. Lucchese and H. G. Koenig, "Religion, spirituality and cardiovascular disease: research, clinical implications, and opportunities in Brazil," Brazilian Journal of Cardiovascular Surgery, vol. 28, no. 1, pp. 103-128, 2013.

[46] Q. Meng et al., "Effect of religion on hypertension in adult Buddhists and residents in China: A cross-sectional study," Scientific RepoRts, vol. 8, no. 1, pp. 1$11,2018$.

[47] Y. C. Cozier et al., "Religious and spiritual coping and risk of incident hypertension in the Black Women's Health Study," Annals of Behavioral Medicine, vol. 52, no. 12, pp. 989-998, 2018.

[48] W. H. M. D. S. Me, "Cardiology Patient Page Cardiology Patient Page," Circulation, vol. 115, pp. e624-e626, 2007.

[49] P. K. Whelton et al., "Primary prevention of hypertension: clinical and public health advisory from The National High Blood Pressure Education Program," Jama, vol. 288, no. 15, pp. 1882-1888, 2002.

[50] S. M. Hegde and S. D. Solomon, "Influence of physical activity on hypertension and cardiac structure and function," Current hypertension reports, vol. 17, no. 10, p. $77,2015$.

[51] S. N. Blair et al., "Influences of cardiorespiratory fitness and other precursors on cardiovascular disease and all-cause mortality in men and women," Jama, vol. 276, no. 3, pp. 205-210, 1996.

[52] C. Erem, A. Hacihasanoglu, M. Kocak, O. Deger, and M. Topbas, "Prevalence of prehypertension and hypertension and associated risk factors among Turkish adults: Trabzon Hypertension Study," Journal of public health, vol. 31, no. 1, pp. 47-58, 2009.

[53] J. E. Sharman, A. La Gerche, and J. S. Coombes, "Exercise and cardiovascular risk in patients with hypertension," American journal of hypertension, vol. 28, no. 2, pp. 147-158, 2015.

[54] R. Rani, V. Mengi, R. Gupta, and H. Sharma, "Hypertension and its risk factors-a cross sectional study in an urban population of a north Indian District," Public Health Research, vol. 5, no. 3, pp. 67-72, 2015.

[55] J. S. Tabrizi, H. Sadeghi-Bazargani, M. Farahbakhsh, L. Nikniaz, and Z. Nikniaz, "Prevalence and associated factors of prehypertension and hypertension in Iranian population: the Lifestyle Promotion Project (LPP)," PloS one, vol. 11, no. 10, p. e0165264, 2016.

[56] M. Priya and C. Mishra, "Prevalence of hypertension in an area of urban Varanasi, India and its association with selected demographic factors," International Journal of Community Medicine and Public Health, vol. 3, no. 6, p. 1600, 2016. 
[57] A. S. Ingale and J. V. Dixit, "Prevalence of hypertension, awareness \& health seeking behaviour among adults residing in field practice area of urban health training centre, government medical college Aurangabad," National Journal of Community Medicine, vol. 8, no. 1, pp. 31-36, 2017.

\section{Figures}

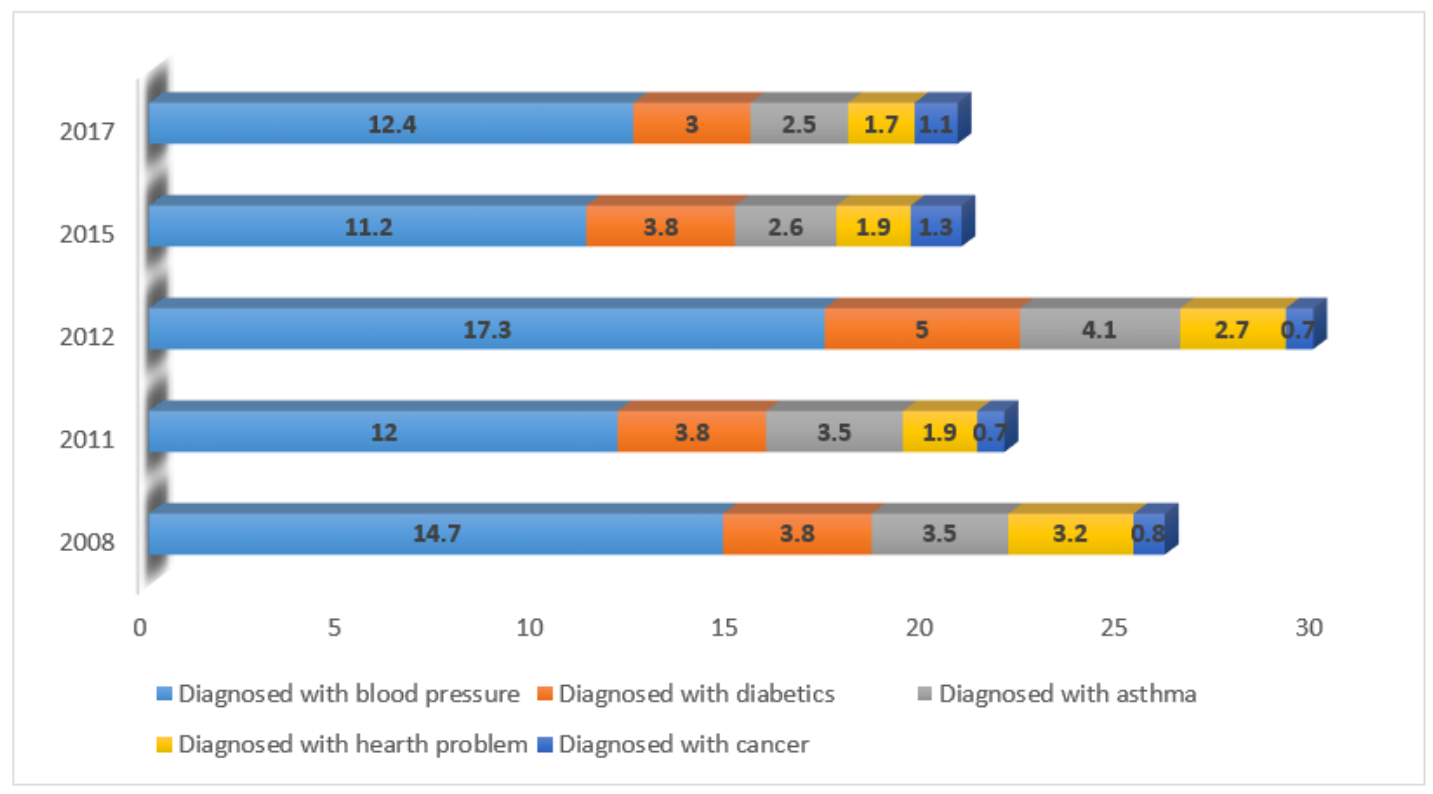

\section{Figure 1}

Trends of non-communicable diseases from 2008-2017

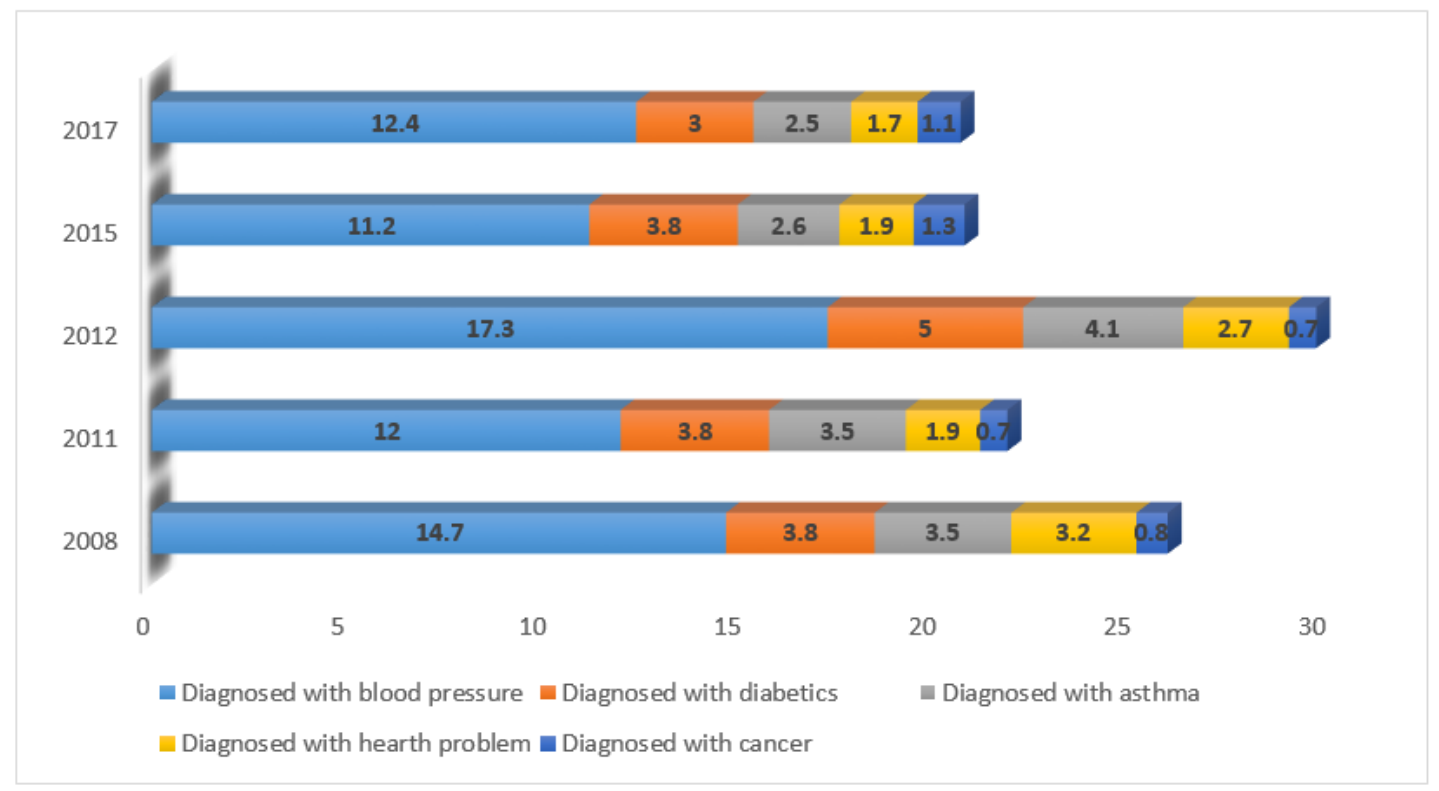

\section{Figure 1}

Trends of non-communicable diseases from 2008-2017 


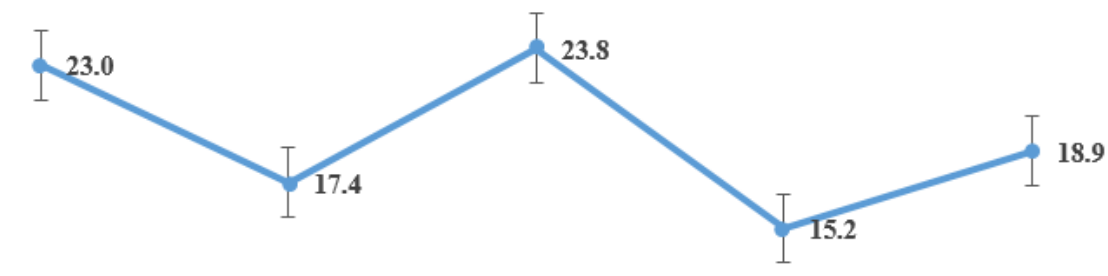

15.0

10.0

5.0

0.0

$2008 \quad 2011$

2012

2015

2017

\section{Figure 2}

Trends of at least one non-communicable disease from 2008-2017

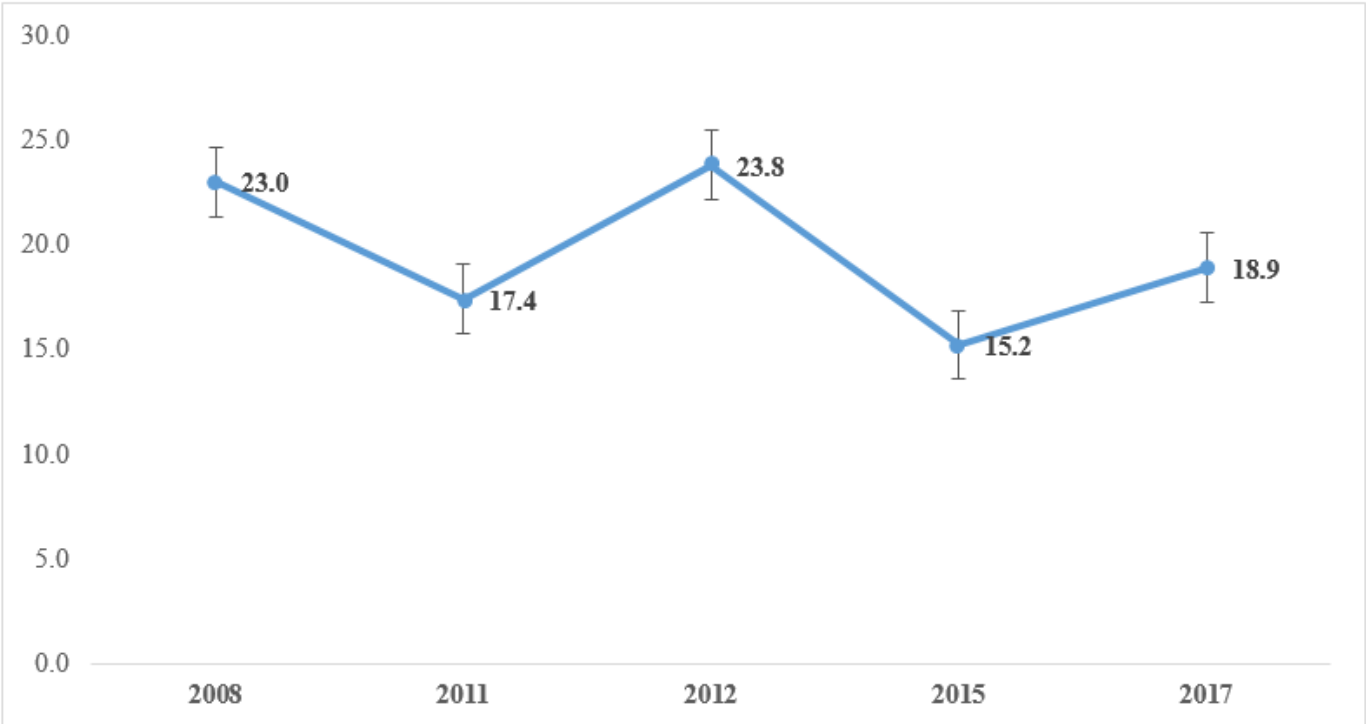

Figure 2

Trends of at least one non-communicable disease from 2008-2017 